\title{
Radial velocities of early-type stars in the Perseus OB2 association ${ }^{\star}$
}

\author{
K. C. Steenbrugge ${ }^{1,2}$, J. H. J. de Bruijne ${ }^{1,3}$, R. Hoogerwerf ${ }^{1,4}$, and P. T. de Zeeuw ${ }^{1}$ \\ 1 Sterrewacht Leiden, Postbus 9513, 2300 RA Leiden, The Netherlands \\ 2 Now at SRON National Institute for Space Research, Sorbonnelaan 2, 3584 CA Utrecht, The Netherlands \\ 3 Now at Astrophysics Missions Division, European Space Agency, ESTEC, Postbus 299, 2200 AG Noordwijk, \\ The Netherlands \\ ${ }^{4}$ Now at Harvard-Smithsonian Center for Astrophysics, 60 Garden Street, MS 31, Cambridge, MA 02138, USA
}

Received 21 January 2003 / Accepted 19 February 2003

\begin{abstract}
We present radial velocities for 29 B- and A-type stars in the field of the nearby association Perseus OB2. The velocities are derived from spectra obtained with AURELIE, via cross correlation with radial velocity standards matched as closely as possible in spectral type. The resulting accuracy is $\sim 2-3 \mathrm{~km} \mathrm{~s}^{-1}$. We use these measurements, together with published values for a few other early-type stars, to study membership of the association. The mean radial velocity (and measured velocity dispersion) of Per OB2 is $23.5 \pm 3.9 \mathrm{~km} \mathrm{~s}^{-1}$, and lies $\sim 15 \mathrm{~km} \mathrm{~s}^{-1}$ away from the mean velocity of the local disk field stars. We identify a number of interlopers in the list of possible late-B- and A-type members which was based on Hipparcos parallaxes and proper motions, and discuss the colour-magnitude diagram of the association.
\end{abstract}

Key words. stars: early-type - stars: binaries: spectroscopic - stars: kinematics - stars: rotation - techniques: radial velocities - open clusters and associations: individual: Perseus OB2

\section{Introduction}

Perseus OB2 is one of the nearest OB associations. It was discovered visually as a loose group of 15 bright, blue stars by Blaauw (1944), who subsequently confirmed his finding by means of radial velocity data from Moore's (1932) catalogue. Blaauw (1952; B52; see also Blaauw 1964) established membership for 17 bright $\mathrm{O}$ and $\mathrm{B}$ stars based on proper motion data. These include a number of well-studied spectroscopic binaries (e.g., AG Per), as well as the high-mass X-ray binary $\mathrm{X}$ Per.

De Zeeuw et al. (1999; Z99) published an updated member list for Per OB2, based on Hipparcos position, proper motion, and parallax data, containing $17 \mathrm{~B}$ - and 16 A-type stars plus a small number of late-type stars. Based on extensive modeling of the kinematics of the Galactic disk, taking selection criteria in the Hipparcos Catalogue into account, Z99 concluded that a significant fraction of the A- and later-type stars identified as astrometric members are likely to be interlopers. This conclusion was confirmed by Belikov et al. (2002a,b), who used Tycho-2 proper motions as well as photometric information. These authors also identified nearly 1000 additional probable

Send offprint requests to: K. C. Steenbrugge, e-mail: K.C.Steenbrugge@sron.nl

* Based on observations made at the Observatoire de Haute-Provence (CNRS), France. members of Per OB2 to 12th magnitude, and suggested that this association may consist of two subgroups.

The Hipparcos satellite (ESA 1997) measured stellar positions, parallaxes, and proper motions, providing fivedimensional data in phase space. The sixth component, the radial velocity, is important, e.g., for improving membership and expansion studies (e.g., Brown et al. 1997). This is particularly true for Per OB2, as the space motion of the association relative to the local disk population is mostly along the line-of-sight and proper motions of member stars are consequently small. Unfortunately, a homogeneous set of radial velocities is not available for the (early-type) Hipparcos members of Per OB2. In this paper, we present new spectroscopic observations of 29 B- and A-type stars in Per OB2.

While determining (relative) spectroscopic radial velocities for late-type stars using cross correlation techniques is feasible with precisions of tens of $\mathrm{m} \mathrm{s}^{-1}$, substantial effort is required to achieve even $\mathrm{km} \mathrm{s}^{-1}$-level precisions for early-type stars (e.g., Verschueren \& David 1999; Verschueren et al. 1999; Griffin et al. 2000). Early-type optical spectra show few absorption lines and these lines are intrinsically broad (up to a few hundred $\mathrm{km} \mathrm{s}^{-1}$ ). They are often broadened even further by stellar rotation and sometimes also show variability due to pulsations and/or stellar winds. The resulting correlation peaks are broad, and can have significant substructure caused by the mixing of spectral lines (cf. Fig. 1 in Verschueren \& David 1999). These 


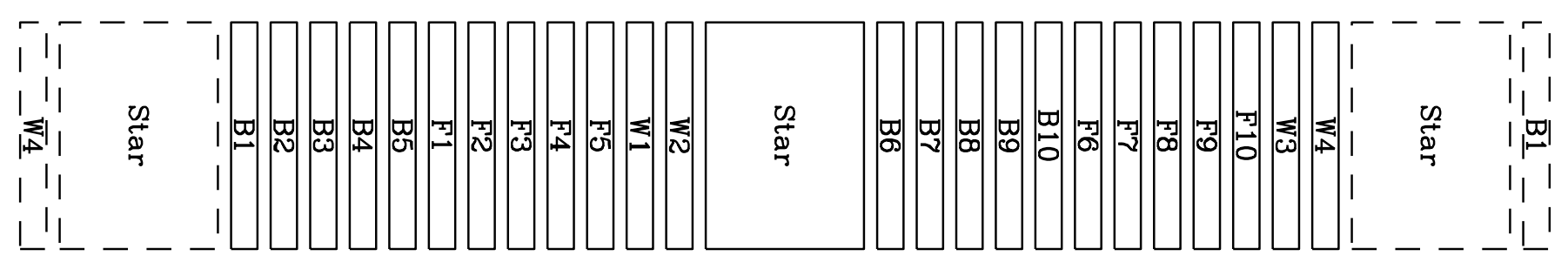

Fig. 1. Observing strategy. Each object exposure (either target or standard star) was preceded by a calibration sequence, consisting of five bias exposures (B1 through B5), five flat field exposures (F1 through F5), and two wavelength calibration arc spectra (W1 and W2), and followed by an identical calibration sequence (B6 through B10, F6 through F10, W3 and W4). In order to minimize overhead, we used the trailing calibration sequence of each star also as the leading sequence for the next star. Time progresses from left to right.

effects complicate accurate centering of correlation peaks and thus the precise determination of (even relative) radial velocities. The derived radial velocities of OBA-type stars, moreover, are known to depend on the spectral region used in the cross correlation (e.g., Verschueren et al. 1999). Furthermore, these stars emit their radiation primarily in the blue part of the spectrum, where CCD devices have reduced quantum efficiencies and slit-centering and atmospheric refraction errors are potentially significant (e.g., Verschueren et al. 1997). Finally, unrecognized multiplicity can be a significant source of error.

We show here that, despite these significant complications, high-resolution spectroscopy combined with a careful observing strategy which includes measurement of many standard stars, does allow measurement of radial velocities for B and A-type stars with an accuracy of a few $\mathrm{km} \mathrm{s}^{-1}$. This makes it possible to improve substantially the membership list for the early-type members of Per OB2. We describe our observations in Sect. 2. In Sect. 3, we focus on the data reduction. The data analysis is presented in Sect. 4. The interpretation of the results and the conclusions are given in Sects. 5 and 6, respectively.

\section{Observations}

The spectra presented here were obtained between Oct. 31 and Nov. 13, 1997, with the $1.52 \mathrm{~m}$ Coudé telescope at Observatoire de Haute-Provence (OHP). We used the AURELIE detector, a linear 2048-pixel photodiode array, which is relatively blue-sensitive (Gillet et al. 1994). We used grating R3 with a linear dispersion of $\sim 16.5 \AA \mathrm{mm}^{-1}$ and a resolving power $R \sim 7000$ in order to achieve a precision of a few $\mathrm{km} \mathrm{s}^{-1}$ in the final radial velocities; this precision is of the same order of magnitude as the typical error in tangential space motions due to Hipparcos proper motion errors at the distance of the Per OB2 association (318 \pm 27 pc; Z99), and comparable to the expected internal velocity dispersion of the group. We used a Th-Ar lamp for wavelength calibration, as well as an image slicer with a circular $3^{\prime \prime}$ entrance aperture, which minimizes potential wavelength errors due to poor centering (Gillet et al. 1994). As our targets are hot B- and A-stars, which primarily show strong hydrogen and helium lines, we used the spectral range from 3800 to $4200 \AA$. This choice resulted in the presence of about 10 higher-order Balmer and helium lines in a typical spectrum. The 3800-4200 $\AA$ spectral region also contains the CaII $\mathrm{H}$ - and K-lines (K: $\lambda=3933 \AA$; H: $\lambda=3968 \AA$; cf. Fig. 3). In B-type stars, the K-line is mostly interstellar, but at spectral types around A0 and later, this line, together with other metallic lines, becomes a notable feature intrinsic to the stellar spectra. For early-type stars, the CaII Hand K-lines thus trace gas along the line of sight instead of stellar kinematics (see, e.g., Sonnentrucker et al. 1999, who actually studied the gas and dust distribution towards Per OB2 using high-resolution spectra of bright stars in the association). Although the presence of (Doppler-shifted) $\mathrm{H}$ - and K-lines can potentially affect cross correlation results and the associated radial velocities (Sect. 4), the CaII metal lines carry minimal weight in practice and their effects are negligibly small.

Z99 established membership of Per OB2 by applying two independent selection methods (the convergent point method of de Bruijne 1999 and the Spaghetti method of Hoogerwerf \& Aguilar 1999) to all Hipparcos entries in a specific field on the sky (see Table A1 in Z99). This approach classified each star as either "certain member" (acceptance by both methods), "possible member" (acceptance by one of the two methods exclusively), or "non-member" (rejection by both methods). As targets for our observations, we selected the 33 B- and A-type certain members of Per OB2 identified by Z99. We added a number of their possible members, as well as some early-type members from B52 which were not confirmed by Z99.

We observed the resulting list in order of decreasing brightness, ultimately obtaining high-quality spectra for 29 distinct targets (Table 1). Exposure times ranged from 10 to $30 \mathrm{~min}$. Many targets were observed multiple times. In total, more than 7 nights of our 2-week observing campaign were weathered out completely.

In addition, we repeatedly obtained high-quality spectra for 20 (candidate) radial-velocity standard stars. As an IAU-approved list of early-type radial velocity standards does not exist, we selected these stars from various sources (Table 2; Sect. 4.1). In this selection, we tried to cover spectral type and luminosity class ranges as large as possible. We also preferentially selected stars with small rotation velocities. Although time consuming, the necessity to build up such a private standard star library, observed with the same instrumental setup as used for the target stars, has the advantage of working with a homogeneous data set, and optimizes the accuracy of the final results (we use "precision" for random and "accuracy" for systematic errors).

We obtained a total of 131 object exposures (target and standard stars together). We decided to take a calibration sequence, consisting of five bias exposures, five flat fields, and 

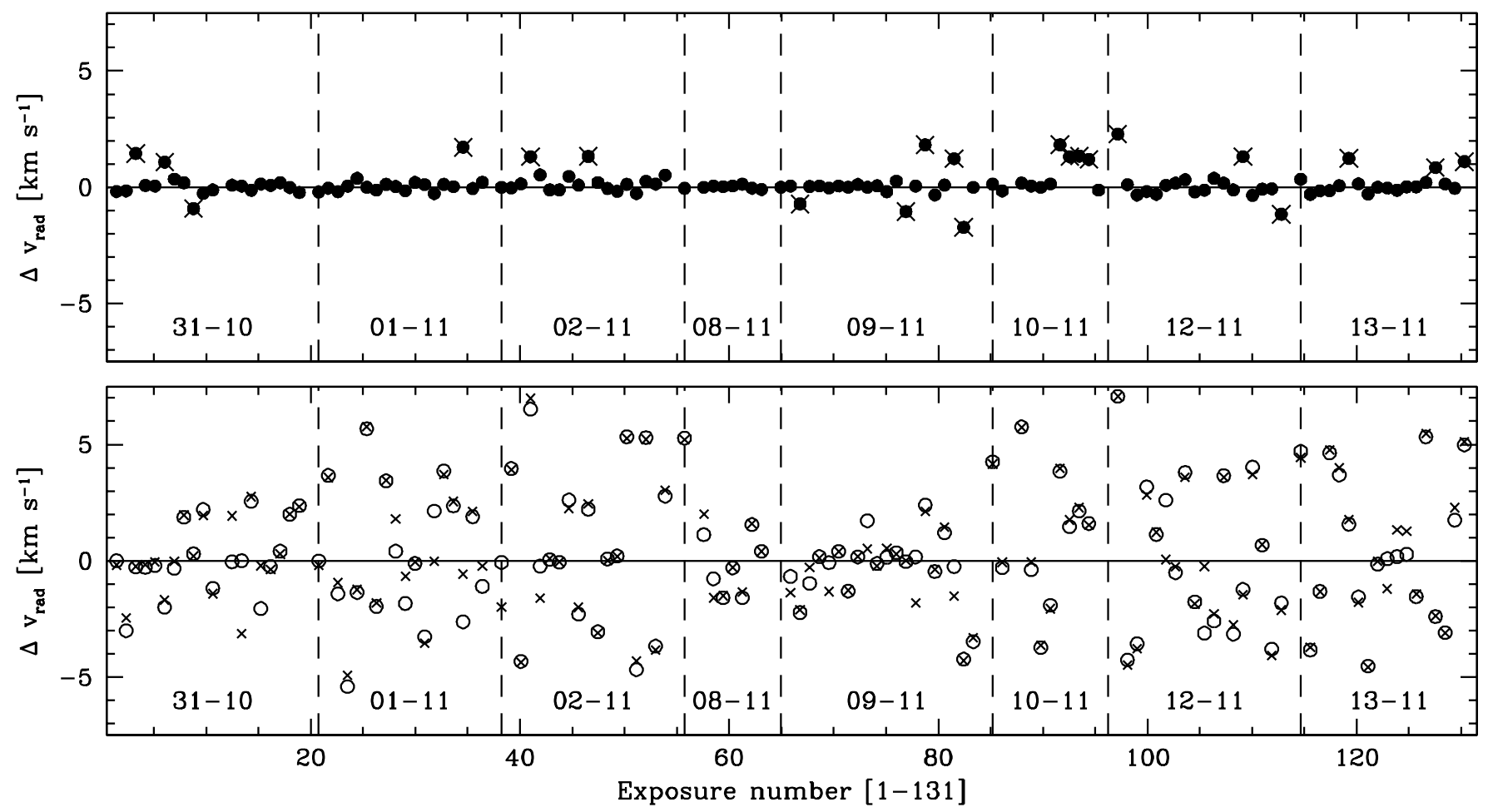

Fig. 2. The stability of the detector, AURELIE. Each of the 131 star exposures has four associated wavelength calibration lamp spectra (W1 through W4): W1 and W2 were obtained before and W3 and W4 after each stellar exposure (Fig. 1). Lamp spectra W3 and W4 were generally taken 30-45 min later than W1 and W2. Top panel: the filled circles show the shift (in $\mathrm{km} \mathrm{s}^{-1}$ ) between the velocity scales inferred from $\mathrm{W} 1$ and from W2. This shift is, for our purposes, acceptably small $\left(\sigma=0.6 \mathrm{~km} \mathrm{~s}^{-1}\right)$, with the exception of 21 cases (crossed symbols). These exposures are treated with extra care in this study. The dashed vertical lines separate different observing nights, the start dates of which are printed in the panel (year: 1997). Bottom panel: open circles and crosses denote the shifts between W1 and W3 and W1 and W4, respectively. Random shifts up to $\sim 10 \mathrm{~km} \mathrm{~s}^{-1}$ occur $\left(\sigma=2.9 \mathrm{~km} \mathrm{~s}^{-1}\right)$, most likely due to instability of the detector (Gillet et al. 1994).

two wavelength calibrations, before and after every star was observed (Fig. 1). In order to reduce overhead, we re-used the trailing calibration sequence of each star as the leading sequence for the next star to be observed. Although this procedure still resulted in a significant overhead, it helped to minimize the effect of instrumental errors in our final results.

\section{Data reduction}

The data reduction was performed using IRAF software (Tody 1993). For each target exposure, we first subtracted the average bias exposure calculated from bias exposures B1 through B10 (Fig. 1). In the next step, we similarly divided by an average flat field exposure. However, we rejected the first of the five flat field exposures in each calibration sequence (F1 and F6; Fig. 1) in order to allow for warming-up effects of the flat field lamp. We then manually removed cosmic rays from our data, after which we wavelength-calibrated the $\mathrm{Th}-\mathrm{Ar}$ lamp spectra.

In order to verify the stability of the detector, we cross correlated the four lamp spectra W1 through W4 taken before and after each star was observed. We found generally good agreement between the wavelength scales inferred from the two lamp spectra taken either before (W1 and W2) or after (W3 and W4) the target exposure $\left(\sigma=0.6\right.$ and $0.7 \mathrm{~km} \mathrm{~s}^{-1}$, respectively; Figs. 1 and 2). However, cross correlating W1 with either W3 or W4, which were taken $\sim 30-45$ min later, showed random shifts of up to $\pm 10 \mathrm{~km} \mathrm{~s}^{-1}\left(\sigma=2.9 \mathrm{~km} \mathrm{~s}^{-1}\right)$. These shifts effectively degrade the resolution of the detector, and are most likely due to thermal instabilities of the detector, which are known to be present at the level of $\sim 0.1$ pixel $\left(\mathrm{hr}^{-1}\right)$ or $\sim 15 \mathrm{~km} \mathrm{~s}^{-1}\left(\mathrm{hr}^{-1}\right)$ (Gillet et al. 1994). Shifts between W1/W2 and W3/W4 cannot be due to telescope re-pointings as AURELIE is located on a dedicated optical bench in the telescope control room. We did not detect any systematic, long-term trend (spanning hours to days) in the detector zero point, consistent with the small repeatability errors derived for a number of late-type stars that were observed throughout our campaign (Sect. 4.3).

Because the lamp spectra in the first calibration sequence (W1 and W2) were taken immediately before the stellar exposure (Fig. 1), we decided not to use the two lamp spectra in the following sequence (W3 and W4), as these were taken after another five bias exposures and flat-field exposures. Unfortunately, even W1 and W2, taken within minutes of each other, show significant shifts $\left(>\sigma=0.6 \mathrm{~km} \mathrm{~s}^{-1}\right)$ in 21 cases (crossed symbols in Fig. 2). We reject the associated exposures in case of a standard star pointing (10 observations; see Col. 5 in Table 2) and treat these exposures with special care in case of a target pointing (11 observations; see Col. 6 in Table 1).

In the final step of the data reduction procedure, we thus wavelength calibrated our spectra using the W1 Th-Ar lamp spectra. Examples of two fully reduced (continuum-subtracted) spectra are shown in Fig. 3. 
Table 1. Observed target stars: B- and A-type members of Per OB2 identified by Z99, supplemented with a number of early-type stars identified as members in pre-Hipparcos investigations (e.g., B52). The stars were observed in order of decreasing brightness, resulting in spectra for 29 distinct targets (62 exposures). Columns: (1) HD number (Hipparcos Catalogue field H71); (2) Hipparcos identifier (H1); (3) name; (4) $V$ magnitude (H5); (5) spectral type and luminosity class; (6) number of spectra obtained ( $N$; subtractions refer to suspect exposures related to detector instability); (7) multiplicity (SB: spectroscopic binary; C: Hipparcos component binary $-\rho$ and $\Delta H p$ in Col. 11; G: Hipparcos acceleration binary; S: Hipparcos suspected non-single - field H61); (8) Hipparcos membership (C: Z99 certain member; P: Z99 possible member; B: Z99 non-member, but B52 member); (9) Hipparcos parallax $\pi$ (H11; mas); (10) Hipparcos parallax error $\sigma_{\pi}$ (H16; mas); (11) Hipparcos components.

\begin{tabular}{|c|c|c|c|c|c|c|c|c|c|c|}
\hline $\begin{array}{l}\mathrm{HD} \\
(1)\end{array}$ & $\begin{array}{l}\mathrm{HIP} \\
(2)\end{array}$ & $\begin{array}{c}\text { Name } \\
(3)\end{array}$ & $\begin{array}{c}V \\
(4)\end{array}$ & $\begin{array}{c}\mathrm{SpT}+\mathrm{LCl} \\
(5)\end{array}$ & $\begin{array}{l}N \\
(6)\end{array}$ & $\begin{array}{l}\text { Mult. } \\
\text { (7) }\end{array}$ & $\begin{array}{r}\text { Z99 } \\
(8)\end{array}$ & $\begin{array}{c}\pi \\
(9)\end{array}$ & $\begin{array}{c}\sigma_{\pi} \\
(10)\end{array}$ & $\begin{array}{l}\text { Hipparcos components } \\
\text { (11) }\end{array}$ \\
\hline 18830 & 14207 & & 8.34 & A0 & 3 & $\mathrm{C}$ & $\mathrm{C}$ & 2.50 & 1.42 & $\rho=0.335, \Delta H p=2.27 \mathrm{mag}$ \\
\hline 19216 & 14450 & & 7.84 & B9V & 3 & & $\mathrm{P}$ & 4.81 & 1.01 & \\
\hline 19567 & 14713 & & 7.62 & B9 & $2-1$ & & $\mathrm{C}$ & 4.54 & 0.96 & \\
\hline 20113 & 15151 & & 7.65 & B8 & $4-1$ & & $\mathrm{P}$ & 3.43 & 0.95 & \\
\hline 20987 & 15895 & & 7.87 & B2V & 2 & G & $\mathrm{C}$ & 1.80 & 1.08 & \\
\hline 21483 & 16203 & & 7.06 & B3III & $2-1$ & & B & 1.60 & 1.05 & \\
\hline 21856 & 16518 & & 5.91 & B1V & 1 & & B & 1.99 & 0.82 & \\
\hline 22114 & 16724 & & 7.60 & $\mathrm{~B} 8 \mathrm{Vp}$ & $2-1$ & & $\mathrm{P}$ & 3.62 & 0.99 & \\
\hline 22951 & 17313 & 40 Per & 4.97 & $\mathrm{~B} 0.5 \mathrm{~V}$ & 1 & S & $\mathrm{C}$ & 3.53 & 0.88 & \\
\hline 23060 & 17387 & & 7.51 & $\mathrm{~B} 2 \mathrm{Vp}$ & 2 & & $\mathrm{P}$ & 2.09 & 0.93 & \\
\hline 23180 & 17448 & o Per & 3.84 & B1III & $3-1$ & $\mathrm{C}+\mathrm{SB}$ & $\mathrm{P}$ & 2.21 & 0.84 & $\rho=1^{\prime \prime} 019, \Delta H p=2.91 \mathrm{mag}$ \\
\hline 23268 & 17498 & & 8.22 & A0 & 3 & & $\mathrm{C}$ & 3.34 & 0.99 & \\
\hline 23478 & 17631 & & 6.68 & B3IV... & 1 & & B & 4.19 & 1.03 & \\
\hline 23597 & 17698 & & 8.19 & B8 & $3-1$ & & $\mathrm{C}$ & 2.56 & 1.01 & \\
\hline 23625 & 17735 & & 6.57 & $\mathrm{~B} 2.5 \mathrm{~V}$ & 1 & $\mathrm{C}+\mathrm{SB}$ & $\mathrm{C}$ & 2.63 & 1.00 & $\rho=3^{\prime \prime} .349, \Delta H p=2.58 \mathrm{mag}$ \\
\hline 23802 & 17845 & & 7.45 & B5Vn & 3 & $\mathrm{C}$ & $\mathrm{C}$ & 3.09 & 1.21 & $\rho=2 . .240, \Delta H p=2.32 \mathrm{mag}$ \\
\hline 24012 & 17998 & & 7.84 & B5 & 2 & & $\mathrm{C}$ & 1.82 & 1.12 & \\
\hline 24131 & 18081 & & 5.78 & $\mathrm{~B} 1 \mathrm{~V}$ & $2-2$ & & $\mathrm{C}$ & 3.15 & 0.84 & \\
\hline 24190 & 18111 & & 7.43 & $\mathrm{~B} 2 \mathrm{~V}$ & $2-1$ & SB & $\mathrm{C}$ & 2.04 & 1.00 & \\
\hline 24398 & 18246 & $\zeta$ Per & 2.84 & $\mathrm{~B} 1 \mathrm{Ib}$ & 1 & & $\mathrm{C}$ & 3.32 & 0.75 & \\
\hline 24583 & 18390 & & 9.00 & B7V & 5 & & $\mathrm{P}$ & 3.31 & 1.35 & \\
\hline 24640 & 18434 & & 5.49 & $\mathrm{~B} 1.5 \mathrm{~V}$ & 1 & S & $\mathrm{P}$ & 3.36 & 0.76 & \\
\hline 24970 & 18621 & & 7.44 & A0 & $3-1$ & & $\mathrm{P}$ & 4.95 & 0.99 & \\
\hline 25539 & 19039 & & 6.87 & B3V & 1 & & $\mathrm{C}$ & 4.19 & 0.97 & \\
\hline 25799 & 19178 & & 7.05 & $\mathrm{~B} 3 \mathrm{~V} \ldots$ & 2 & SB & $\mathrm{C}$ & 2.78 & 0.95 & \\
\hline 25833 & 19201 & AG Per & 6.70 & B5V:p & $1-1$ & $\mathrm{C}+\mathrm{SB}$ & $\mathrm{C}$ & 3.89 & 1.31 & $\rho=0 .{ }^{\prime} 803, \Delta H p=1.81 \mathrm{mag}$ \\
\hline 26499 & 19659 & & 9.06 & B9 & 2 & & $\mathrm{C}$ & 4.14 & 1.30 & \\
\hline 278942 & 17113 & & 9.03 & B3III & 2 & $\mathrm{C}$ & $\mathrm{C}$ & 4.83 & 1.21 & $\rho=0.149, \Delta H p=0.78 \mathrm{mag}$ \\
\hline 281159 & 17465 & & 8.51 & B5V & 2 & $\mathrm{C}$ & $\mathrm{P}$ & 4.52 & 3.30 & $\rho=0 . ' 615, \Delta H p=0.25 \mathrm{mag}$ \\
\hline
\end{tabular}

\section{Data analysis}

Radial velocities are generally determined by means of spectral cross correlation between the target and a suitable standard star (but see, e.g., Dravins et al. 1999). Standard star spectra can either be obtained observationally or generated by means of stellar evolutionary codes (e.g., Morse et al. 1991). Whereas the latter approach has the advantage that the spectral type, luminosity class, rotation velocity, etc. of the template can be chosen representative of the target spectrum, significant uncertainties exist in the (atmospheric) models of early-type stars (e.g., Lennon et al. 1992). We chose to use our own standard star spectra for radial velocity calibration. Possible drawbacks in this approach are, e.g., unrecognized multiplicity and errors in catalogued radial velocities and/or spectral classifications.

\subsection{Standard stars}

Our 20 early-type standard stars have been studied carefully in the past, mostly with the aim of establishing binarity, and they have never shown signs of velocity variability, although HD 27638, HD 196724, and HD 214994 may have astrometric companions (Table 2). Selection criteria in the construction of this standard star sample included visibility, visual magnitude, spectral type, luminosity class, and small rotational velocity.

The published radial velocities for our standard stars generally agree to within $\sim 1 \mathrm{~km} \mathrm{~s}^{-1}$, but differences up to $\sim 3 \mathrm{~km} \mathrm{~s}^{-1}$ occur. Some of these discrepancies may be due to differences in the absolute zero points used in different studies. In order to minimize the effect of this uncertainty, we preferentially selected the radial velocities for our standard stars from 

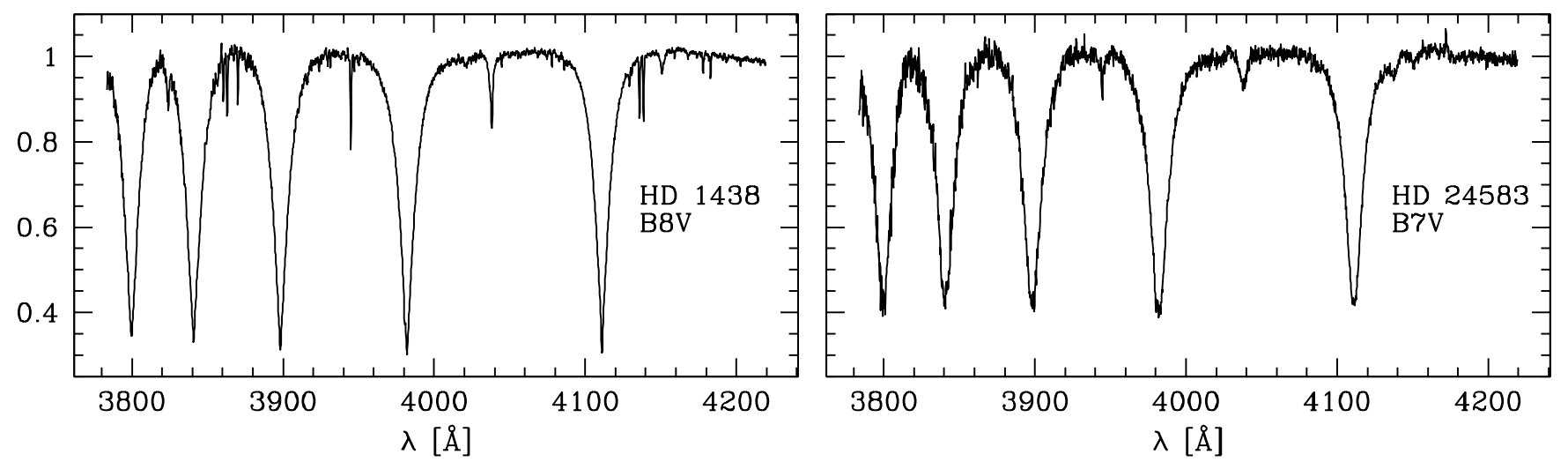

Fig. 3. Example of reduced (continuum-subtracted) spectra: HD 1438 (B8V; $V=6.10 \mathrm{mag} ; T=30 \mathrm{~min}$; left panel) and HD 24583 (B7V; $V=9.00 \mathrm{mag} ; T=30 \mathrm{~min}$; right panel). Note the near-absence of metal lines, the broad nature of the higher-order Balmer hydrogen lines and the helium lines, and the spectral differences in the helium lines.

Table 2. Stars used as radial velocity standards. Columns: (1) HD number (Hipparcos Catalogue field H71); (2) Hipparcos identifier (H1); (3) $V$ magnitude (H5); (4) spectral type and luminosity class (H76); (5) number of spectra obtained ( $N$; subtractions refer to rejected exposures due to detector instability); (6) multiplicity (G: Hipparcos acceleration binary; S: Hipparcos suspected non-single - field H61) (7) assumed radial velocity $\left(\mathrm{km} \mathrm{s}^{-1}\right.$; see Sect. 4.1 for details); (8) idem from Morse et al. (1991; Table 6; Table 4 for HD 23408); (9) radial velocity in km s ${ }^{-1}$ from Fekel (1985; Table 3); (10) idem from Wolff (1978; range <3 · standard deviation; Tables 1 and 3); (11) idem from Abt \& Levy (1978; Table 1); (12) idem from Gies \& Bolton (1986; Table 3); (13) idem from Latham \& Stefanik (1982; Table 1).

\begin{tabular}{|c|c|c|c|c|c|c|c|c|c|c|c|c|}
\hline \multirow{2}{*}{$\begin{array}{l}\text { HD } \\
\text { (1) }\end{array}$} & \multirow{2}{*}{$\begin{array}{l}\text { HIP } \\
(2)\end{array}$} & \multirow{2}{*}{$\begin{array}{c}V \\
(3)\end{array}$} & \multirow{2}{*}{$\begin{array}{c}\mathrm{SpT}+\mathrm{LCl} \\
\text { (4) }\end{array}$} & \multirow{2}{*}{$\begin{array}{l}N \\
(5)\end{array}$} & \multirow{2}{*}{$\begin{array}{l}\text { Mult. } \\
\text { (6) }\end{array}$} & \multicolumn{7}{|c|}{ Radial velocity $\left[\mathrm{km} \mathrm{s}^{-1}\right]$} \\
\hline & & & & & & (7) & (8) & $(9)$ & $(10)$ & (11) & (12) & (13) \\
\hline 1438 & 1501 & 6.10 & B8V & 3 & & +3.3 & & . & . & . & . & +3.3 \\
\hline 3360 & 2920 & 3.69 & B2IV & $3-1$ & & -0.3 & -0.3 & . & . & +1.0 & . & +0.5 \\
\hline 10982 & 8387 & 5.86 & B9V & $3-1$ & & +5.0 & & . & +5.0 & . & . & . \\
\hline 17081 & 12770 & 4.24 & B7IV & $2-1$ & & +15.4 & +15.4 & +14.3 & . & . & . & . \\
\hline 23408 & 17573 & 3.87 & B8III & 2 & & +6.5 & +6.5 & . & . & . & . & +7.6 \\
\hline 26912 & 19860 & 4.27 & B3IV & $2-1$ & & +14.9 & +14.9 & . & . & +15.5 & . & . \\
\hline 27638 & 20430 & 5.38 & B9V & 2 & $S$ & +14.4 & . & . & 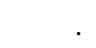 & 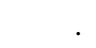 & . & +14.4 \\
\hline 28114 & 20715 & 6.06 & B6IV & $2-1$ & & +12.9 & 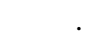 & 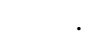 & +12.9 & . & . & +13.4 \\
\hline 35708 & 25539 & 4.88 & B2.5IV & 2 & & +17.0 & +17.0 & +18.1 & 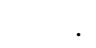 & +15.2 & . & +18.4 \\
\hline 36267 & 25813 & 4.20 & B5V & $3-1$ & & +19.8 & 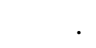 & & 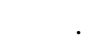 & +19.8 & . & . \\
\hline 38899 & 27511 & 4.89 & B9IV & 2 & & +21.6 & +21.6 & +21.6 & +22.2 & 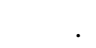 & . & +20.9 \\
\hline 43112 & 29678 & 5.91 & B1V & 2 & & +37.3 & +37.3 & 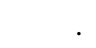 & 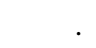 & . & +35.8 & . \\
\hline 58142 & 36145 & 4.61 & A1V & $5-1$ & & +26.0 & +26.0 & +27.2 & . & . & 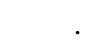 & +26.9 \\
\hline 196724 & 101867 & 4.81 & A0V & $6-1$ & G & $-18.4^{a}$ & -12.0 & & 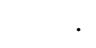 & . & . & . \\
\hline 196821 & 101919 & 6.08 & A0III & $4-1$ & & -31.3 & 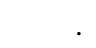 & -31.3 & -31.6 & . & . & . \\
\hline 201345 & 104316 & 7.78 & O9p & 1 & & +21.6 & . & 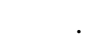 & 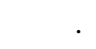 & . & +21.6 & . \\
\hline 214994 & 112051 & 4.80 & A1IV & 5 & G & +9.1 & +9.1 & +7.5 & . & . & 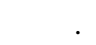 & +8.5 \\
\hline 217811 & 113802 & 6.37 & B2V & 3 & & -11.2 & -11.2 & . & . & . & 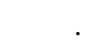 & -10.2 \\
\hline 219188 & 114690 & 7.06 & B0.5III & $4-1$ & & $+48.0^{b}$ & 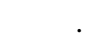 & . & 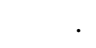 & . & +68.0 & . \\
\hline 220599 & 115591 & 5.56 & B9III & 2 & & +12.0 & . & . & +12.0 & . &. & \\
\hline
\end{tabular}

${ }^{a}$ Fekel 1990 (cf. Liu et al. 1989).

${ }^{b}$ SIMBAD.

the source containing most measurements in absolute numbers (Morse et al. 1991; results based on CCD data). For the remaining stars, we used, in order of decreasing preference, Fekel (1985), Wolff (1978), Abt \& Levy (1978), Gies \& Bolton (1986), and Latham \& Stefanik (1982). Exceptions to this rule were made for HD 196724 and 219188, for which we assume a radial velocity of $-18.4 \mathrm{~km} \mathrm{~s}^{-1}$ (Fekel 1990; Liu et al. 1989) and $+48.0 \mathrm{~km} \mathrm{~s}^{-1}$ (SIMBAD), respectively. Upon comparing the accordingly selected radial velocities with the values contained in the compilation catalogue of mean radial velocities of
Barbier-Brossat \& Figon (2000), we find a mean difference of $0.1 \mathrm{~km} \mathrm{~s}^{-1}$ and a standard deviation of $2.0 \mathrm{~km} \mathrm{~s}^{-1}$. These values (probably) mostly reflect zero-point differences (and random errors) and imply the accuracy and precision of our final radial velocities are $\gtrsim 0.1 \mathrm{~km} \mathrm{~s}^{-1}$ and $\gtrsim 2.0 \mathrm{~km} \mathrm{~s}^{-1}$, respectively.

\subsection{Cross correlation}

We cross correlated the spectra by means of the standard IRAF task fxcor. In order to suppress noise, we applied 
high-frequency Fourier filtering to all spectra (cf. Verschueren \& David 1999). We did not apply low-pass filtering, and used a Gaussian fitting function for centering of the correlation peak. This function was found empirically to be a suitable choice. The detector instability did not justify a more refined fitting function. In order to suppress the effect of continuum mismatch, we subtracted the continuum from both the template and the target spectrum - and thus normalized both spectra to a continuum level of 1 - before each cross correlation.

\subsection{Repeatability errors}

We observed many of the objects, either target or standard stars, multiple times. Under the assumption that these stars are single, cross correlation of their spectra (on an object-by-object and exposure-by-exposure basis) should ideally result in vanishingly small shifts. Conversely, shifts measured in such an exercise provide a direct estimate of the random errors that will be present in the final radial velocities (e.g., Morse et al. 1991; Verschueren et al. 1997). In our case, these random errors include the temporal instability of the detector (Sect. 3).

Figure 4 shows the repeatability errors inferred from our data, after excluding known spectroscopic binaries. We define the repeatability error $\sigma_{\mathrm{r}}$ for a pair of exposures of a given object as the relative shift (in $\mathrm{km} \mathrm{s}^{-1}$ ) emerging during their cross correlation. Sixteen of our standard stars and 18 of our Per OB2 targets were observed multiple times, resulting in 86 repeatability errors (the two exposures of the faint target HD 278942 lead to a repeatability error of $\sim 16 \mathrm{~km} \mathrm{~s}^{-1}$; as these spectra are noise-dominated, we reject this object from this discussion). From Fig. 4 we conclude that the repeatability errors are at the level of $\sim 0-3 \mathrm{~km} \mathrm{~s}^{-1}$, consistent with the expected noise due to thermal instabilities of the detector (Fig. 2). The mean repeatability error is $\overline{\sigma_{\mathrm{r}}}=2.1 \mathrm{~km} \mathrm{~s}^{-1}$ (the median is $2.2 \mathrm{~km} \mathrm{~s}^{-1}$ ); this value could be slightly overestimated as a result of unrecognized multiplicity. A value of $2.1 \mathrm{~km} \mathrm{~s}^{-1}$ is comparable to repeatability errors quoted in the literature (e.g., Morse et al. 1991: 1-3 km s${ }^{-1}$; Verschueren et al. 1997: 0.7-1.4 $\mathrm{km} \mathrm{s}^{-1}$ ).

We also repeatedly observed two late-type stars (HD 18449, K2III, $N=7$; HD 219615, G7III, $N=4$ ). The repeatability errors for these stars are small, 0.6 and $0.9 \mathrm{~km} \mathrm{~s}^{-1}$, respectively, partly reflecting the relative ease with which late-type spectra can be cross correlated. The $N=7$ exposures of HD 18449 were observed on 7 different nights; the low value of the corresponding repeatability error is consistent with the absence of significant night-to-night instrumental zero-point shifts (Sect. 3 and Fig. 2).

\subsection{Template (mis)match: Standard star selection}

A radial velocity determination by means of cross correlation of an observed spectrum with that of a velocity standard can be biased if the spectrum of the target does not have exactly the same characteristics as that of the standard star. This template mismatch can, e.g., be due to differing rotational velocities (Sect. 4.5). It can also result from spectral type and/or luminosity class differences, which may translate into differences

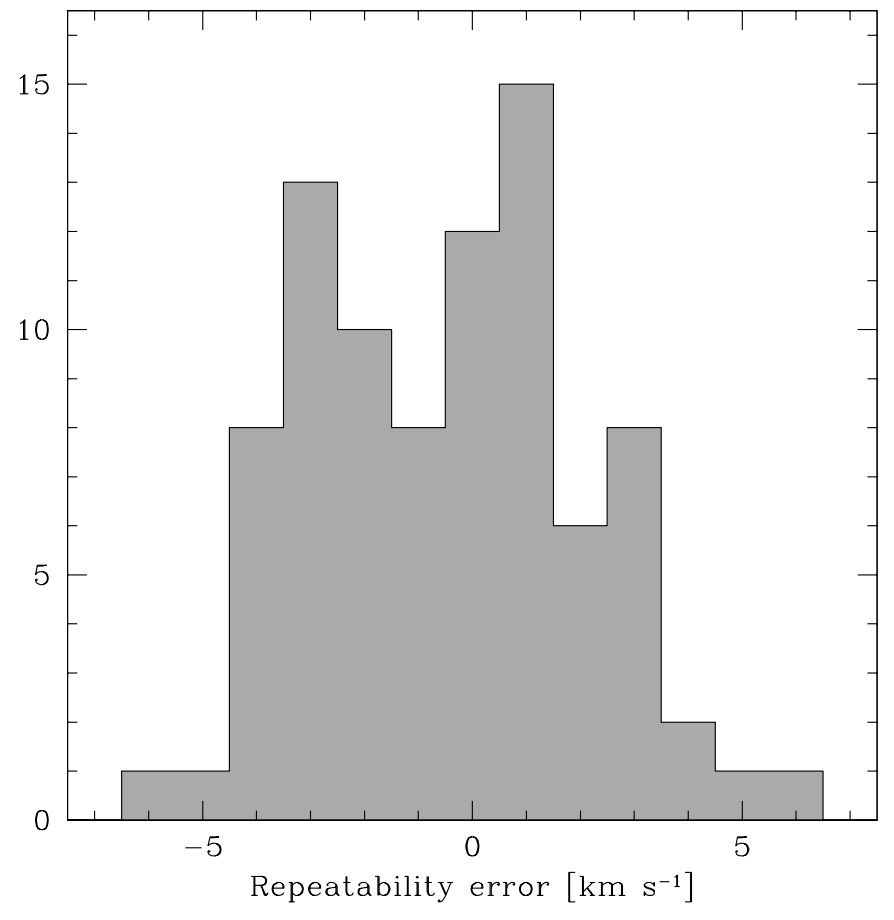

Fig. 4. Repeatability errors for 86 pairs of spectra (either target or standard stars; see Sect. 4.3 for details). In the remainder of this paper, we assume that repeatability errors $\left(\sigma_{\mathrm{r}}\right)$ are positive. The mean and median repeatability errors are 2.1 and $2.2 \mathrm{~km} \mathrm{~s}^{-1}$, respectively.

in atmospheric velocity fields (convection, stellar wind, pulsation, ...), in line blending, line blanketing, line asymmetries (due to Stark broadening), or in stellar continuum.

We decided to use Hipparcos spectral classifications (catalogue fields $\mathrm{H} 76$ and H77) throughout this study. These values are extracted from ground-based compilations, mainly SIMBAD and the Michigan Spectral Survey (see ESA 1997 for details), and do not necessarily have a high and/or homogeneous quality. Classification errors (e.g., at subclass level) can thus not be excluded. In case the Hipparcos spectral classification lacked a luminosity class, we assumed the star was a dwarf. We ignored classification details/peculiarities such as "p", "n", and "e". Comparing the Hipparcos spectral types and luminosity classes with recent SIMBAD values and/or other literature values did not reveal significant differences, except for the following target stars: HD 20987 (Hipparcos: B9 $\Rightarrow$ Abt 1985: B2V), HD 23802 (Hipparcos: B9 $\Rightarrow$ Guetter 1977: B5Vn), HD 24012 (Hipparcos: B9 $\Rightarrow$ Blaauw \& van Albada 1963: B5), HD 24583 (Hipparcos: A0 $\Rightarrow$ Guetter 1977: B7V), and HD 278942 (Hipparcos: F2 $\Rightarrow$ Cernis 1993: B3III). We discuss these stars individually in Sect. 4.8. We decided, for these five "problem cases", to use the spectral classifications derived in the dedicated studies listed above. Alternatively, we could have used, e.g., the strength of the helium lines in our spectra as a coarse spectral type indicator (for example, the ratio HeI 4471/MgII 4481, not accessible from our data, is often used for determining the spectral types of stars; Jaschek \& Jaschek 1990). In case of line anomalies, line indicators may provide misleading classifications. B-type stars, and notably the helium lines in their spectra, are known to be 


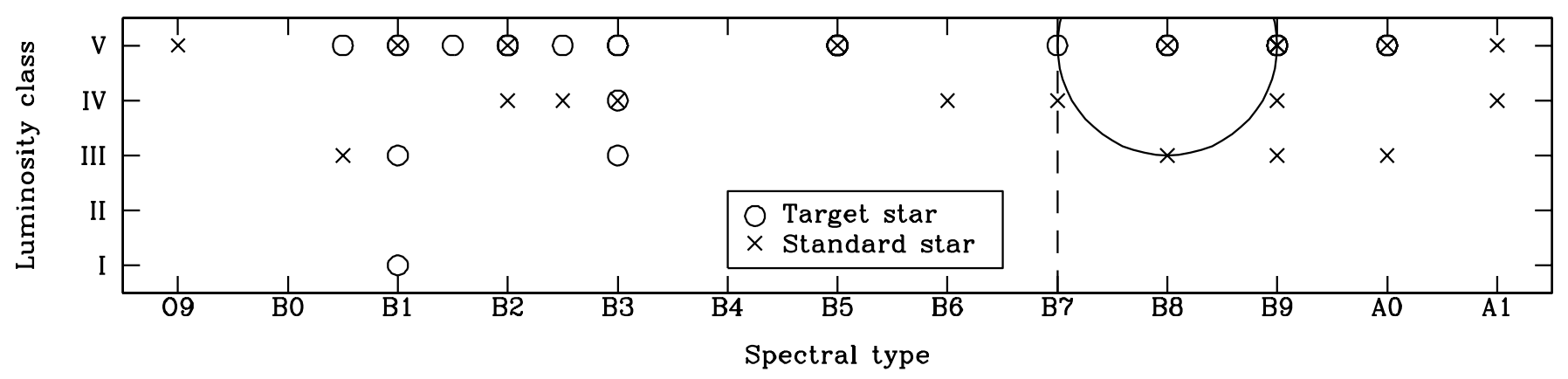

Fig. 5. Luminosity classes (LCl) and spectral types (SpT) of target stars (open circles) and standard stars (crosses). This diagram is used for selection of suitable standard stars for cross correlation. For a given target star with luminosity class $\mathrm{LCl}_{*}$ and spectral type $\mathrm{SpT}$, we consider all $N_{\mathrm{s}}$ standard stars for which $d \leq d_{\max }$ (Eq. (1)). For spectral types later than B7 (>B7; dashed vertical line), we use $d_{\max }=1.0$ while for stars with $\mathrm{SpT}_{*}=\mathrm{B} 7$ or earlier, we use $d_{\max }=1.5$ because of sparsity of (observed) standard stars. The circle demonstrates the selection of standard stars for a target star with $\mathrm{SpT}_{*}=\mathrm{B} 8$ and $\mathrm{LCl}_{*}=\mathrm{V}$; for this object, we consider $N_{\mathrm{s}}=3$ velocity standards as appropriate templates (the three crosses falling within the circle of radius 1). The final radial velocity $v_{\text {rad }}$ for this target star (Eq. (3); Table 3) is obtained by means of a distance-weighted average of the individual radial velocities $v_{\mathrm{rad}, i}$ obtained using all $N_{\mathrm{e}}$ exposures of the $i=1, \ldots, N_{\mathrm{s}}=3$ standard stars (Sect. 4.4).

particularly sensitive to these problems. Dozens of peculiar stars, for example in the form of helium-strong and heliumweak stars, have been discovered in the nearby OB associations (Jaschek \& Jaschek 1990), and we therefore decided not to use helium line strengths as spectral type indicators.

In order to investigate the effect of spectral mismatch (including luminosity class mismatch), we performed a cross correlation of all possible combinations of all exposures of all 20 standard stars. After averaging over exposure pairs, we constructed a $20 \times 20$ "matrix" of average shifts between all pairs of standard stars. The diagonal elements in this matrix, which is skew-symmetric, correspond to the repeatability errors (Sect. 4.3). After ordering the stars on spectral type, one can see that shifts grow when moving away from the diagonal. However, close to the diagonal (i.e., within a few matrix elements, i.e., generally within a few sub-classes), shifts are mostly relatively small (they are even generally insignificant in this exercise as a result of random errors). The results of this analysis indicate, nonetheless, that spectral mismatch can easily give rise to $5-10 \mathrm{~km} \mathrm{~s}^{-1}$ systematic errors or larger. For this reason, we decided, for a given target star exposure, not to use a single radial velocity standard as template. Instead, we used a set of standard stars with spectral types and luminosity classes "similar to" the target, and averaged the corresponding radial velocities to one, final value. This has the additional advantage that radial velocity zero-point differences/errors in our standard star sample are washed out to some degree.

In order to quantify the meaning of "similar spectral types and luminosity classes", we defined a "distance" $d$ between any standard and any target star:

$d \equiv \sqrt{\left(\mathrm{SpT}_{*}-\mathrm{SpT}\right)^{2}+\frac{1}{4} \cdot\left(\mathrm{LCl}_{*}-\mathrm{LCl}\right)^{2}}$.

We then constructed a luminosity class-spectral type (LClSpT) diagram containing all target and standard stars (Fig. 5). From this diagram, we decided to define all standard stars for which $d \leq d_{\max }$ as suitable templates for a target star with luminosity class $\mathrm{LCl}_{*}$ and spectral type $\mathrm{SpT}_{*}$. The unit of the distance $d$ was chosen such that a difference of one spectral type subclass and a difference of two luminosity classes both denote a distance of 1 (e.g., "B8 - B6 $\rightarrow d=2$ " and "V - III $\rightarrow d=1$ "). The maximum distance $\left(d_{\max }\right)$ was chosen as small as possible, but still large enough for most target stars to have more than one associated standard star:

$d_{\max }= \begin{cases}1.5 & \text { for } \mathrm{SpT}_{*} \leq \mathrm{B} 7 \\ 1.0 & \text { for } \mathrm{SpT}_{*}>\mathrm{B} 7\end{cases}$

where $\leq$ B7 means B7 or earlier and $>$ B7 means later than B7. The radial velocity $v_{\text {rad }}$ for the target is obtained by means of a distance-weighted average of the individual radial velocities $v_{\mathrm{rad}, i}$ obtained by cross correlating with all $N_{\mathrm{e}}$ exposures of the $i=1, \ldots, N_{\mathrm{s}}$ suitable standard stars:

$v_{\mathrm{rad}}=\frac{\sum_{i=1}^{N_{\mathrm{e}}} w_{i} \cdot v_{\mathrm{rad}, i}}{\sum_{i=1}^{N_{\mathrm{e}}} w_{i}}$

where

$w_{i}=d_{\max }-0.5 \cdot d_{i}$.

This choice for $w_{i}$ has the property that $w_{i}\left(d_{i}=0\right) / w_{i}\left(d_{i}=\right.$ $\left.d_{\max }\right)=2$, independent of spectral type.

As an example, Fig. 3 shows the spectra of HD 1438 and HD 24583; the first star (B8V) has been used as a standard in the radial velocity determination of the latter (B7V). Although the signal-to-noise ratios and rotational velocities of the two spectra/stars differ, minor spectral differences are clearly visible, e.g., in the helium line at $4026 \AA$. The "spectraltype-distance" $d$ between the stars equals 1 (Eq. (1)), which is the maximum allowed distance $\left(d_{\max }\right)$ for targets with spectral types B7V and later.

\subsection{Template (mis)match: Stellar rotation}

We carried out a simple experiment to investigate the effect of rotational mismatch on the final radial velocities. We selected the $\mathrm{H} \delta$ absorption line in the spectrum of the slow rotator HD 38899 (B9IV; $v \sin i=15-30 \mathrm{~km} \mathrm{~s}^{-1}$ ). We broadened 
Table 3. Radial velocities for 29 target stars. Each star occupies $N$ lines, with $N$ the number of spectra/exposures obtained (Table 1). Columns: (1) HD number; (2) spectral type and luminosity class; (3) multiplicity (SB: spectroscopic binary; C: Hipparcos component binary; G: Hipparcos acceleration binary; S: Hipparcos suspected non-single - field H61); (4) the number of suitable standard stars $N_{\mathrm{s}}$ and, between brackets, the associated total number of exposures $N_{\mathrm{e}}$ (Sect. 4.4); (5) the average distance to the $N_{\mathrm{s}}$ standard stars (Sect. 4.6); (6) exposure number $(1, \ldots, N$; asterisks denote suspect exposures related to detector instability - see Sect. 3 for details); (7) heliocentric Julian date of the mid-point of the exposure (HJD 24507XX.XXXXX); (8) final distance-weighted radial velocity $v_{\text {rad }}\left(\mathrm{km} \mathrm{s}^{-1}\right.$; Eq. (3) in Sect. 4.4); (9) corresponding standard deviation $\sigma_{v, \text { rad }}\left(\mathrm{km} \mathrm{s}^{-1}\right.$; Sect. 4.6); (10) time-averaged radial velocity $\bar{v}_{\text {rad }}$ using the $N$ exposures $\left(\mathrm{km} \mathrm{s}^{-1}\right.$; spectroscopic binaries show a dash; Sect. 4.6); (11) corresponding standard deviation $\sigma_{\bar{v}, \text { rad }}\left(\mathrm{km} \mathrm{s}^{-1}\right.$; values smaller than $2.0 \mathrm{~km} \mathrm{~s}^{-1}$ are optimistic; Sect. 4.6); (12) literature radial velocity $\left(\mathrm{km} \mathrm{s}^{-1}\right.$ ); (13) source of literature radial velocity (Col. 12) and remarks (B52: Blaauw 1952; BvA: Blaauw \& van Albada 1963; Z83: Zentelis 1983).

\begin{tabular}{|c|c|c|c|c|c|c|c|c|c|c|c|c|}
\hline $\begin{array}{l}\text { HD } \\
(1)\end{array}$ & $\begin{array}{l}\mathrm{SpT} \\
(2)\end{array}$ & $\begin{array}{l}\text { Mult. } \\
\text { (3) }\end{array}$ & $\begin{array}{c}N_{\mathrm{s}}\left(N_{\mathrm{e}}\right) \\
(4)\end{array}$ & $\begin{array}{c}\bar{d} \\
(5)\end{array}$ & $\begin{array}{c}\text { Expos. } \\
\text { (6) }\end{array}$ & $\begin{array}{l}\text { HJD } \\
(7)\end{array}$ & $\begin{array}{l}v_{\mathrm{rad}} \\
(8)\end{array}$ & $\begin{array}{c}\sigma_{v, \mathrm{rad}} \\
(9)\end{array}$ & $\begin{array}{l}\bar{v}_{\text {rad }} \\
(10)\end{array}$ & $\begin{array}{c}\sigma_{\bar{\nabla}, \mathrm{rad}} \\
(11)\end{array}$ & $\begin{array}{c}v_{\text {lit }} \\
(12)\end{array}$ & $\begin{array}{l}\text { Remark } \\
(13)\end{array}$ \\
\hline \multirow[t]{3}{*}{18830} & $\mathrm{~A} 0$ & $\mathrm{C}$ & $5(16)$ & 0.69 & 1 & 61.34014 & 5.1 & 3.2 & 3.8 & 1.5 & $10.5 \pm 5.6$ & Grenier et al. (1999) \\
\hline & & & $5(16)$ & 0.69 & 2 & 61.36894 & 2.2 & 3.4 & & & & \\
\hline & & & $5(16)$ & 0.69 & 3 & 61.39773 & 4.1 & 3.3 & & & & \\
\hline \multirow[t]{3}{*}{19216} & B9V & & $6(16)$ & 0.69 & 1 & 66.49641 & 7.8 & 3.4 & 8.9 & 1.3 & $4.5 \pm 2.7$ & Grenier et al. (1999) \\
\hline & & & $6(16)$ & 0.69 & 2 & 66.52524 & 10.3 & 3.5 & & & & \\
\hline & & & $6(16)$ & 0.69 & 3 & 66.55355 & 8.7 & 3.6 & & & & \\
\hline \multirow[t]{2}{*}{19567} & B9 & & $6(16)$ & 0.69 & 1 & 54.62964 & 1.7 & 3.6 & 2.6 & 1.3 & & \\
\hline & & & $6(16)$ & 0.69 & $2 *$ & 54.65873 & 3.5 & 3.8 & & & & \\
\hline \multirow[t]{4}{*}{20113} & B8 & & $4(9)$ & 0.67 & 1 & 66.58191 & 6.1 & 4.7 & 6.2 & 2.4 & & \\
\hline & & & $4(9)$ & 0.67 & 2 & 66.61018 & 4.3 & 4.8 & & & & \\
\hline & & & $4(9)$ & 0.67 & $3 *$ & 66.63902 & 4.7 & 5.2 & & & & \\
\hline & & & $4(9)$ & 0.67 & 4 & 66.66758 & 9.5 & 4.3 & & & & \\
\hline \multirow[t]{2}{*}{20987} & $\mathrm{~B} 2 \mathrm{~V}$ & G & $3(7)$ & 0.34 & 1 & 54.68775 & -22.6 & 5.7 & -22.1 & 0.8 & & SpT from Abt (1985) \\
\hline & & & $3(7)$ & 0.34 & 2 & 54.71652 & -21.5 & 4.5 & & & & \\
\hline \multirow[t]{2}{*}{21483} & B3III & & $4(8)$ & 1.05 & 1 & 66.36154 & -4.0 & 3.5 & -3.6 & 0.5 & $-6.0 \pm 0.5$ & B52 \\
\hline & & & $4(8)$ & 1.05 & $2^{*}$ & 66.39006 & -3.2 & 3.5 & & & & \\
\hline 21856 & B1V & & $4(10)$ & 0.86 & 1 & 65.36623 & 29.7 & 3.3 & 29.7 & 3.3 & $31.5 \pm 3.0$ & Z83 \\
\hline \multirow[t]{2}{*}{22114} & B8Vp & & $4(9)$ & 0.67 & 1 & 65.67265 & 4.7 & 3.2 & 6.1 & 1.9 & & \\
\hline & & & $4(9)$ & 0.67 & $2 *$ & 65.70122 & 7.4 & 3.4 & & & & \\
\hline 22951 & B $0.5 \mathrm{~V}$ & $S$ & $4(9)$ & 1.11 & 1 & 62.38453 & 19.3 & 6.3 & 19.3 & 6.3 & $19.6 \pm 3.0$ & Z83 \\
\hline \multirow[t]{2}{*}{23060} & $\mathrm{~B} 2 \mathrm{Vp}$ & & $5(10)$ & 0.55 & 1 & 65.41966 & 19.2 & 4.0 & 21.1 & 2.6 & $28.5 \pm 0.9$ & BvA \\
\hline & & & $5(10)$ & 0.55 & 2 & 65.44917 & 22.9 & 4.0 & & & & \\
\hline \multirow[t]{3}{*}{23180} & B1III & $\mathrm{C}+\mathrm{SB}$ & $4(10)$ & 1.00 & $1^{*}$ & 63.61336 & -6.1 & 3.3 & - & - & $12.2 \pm 0.5$ & Stickland \& Lloyd (1998) \\
\hline & & & $4(10)$ & 1.00 & 2 & 63.65607 & 6.4 & 3.1 & & & & \\
\hline & & & $4(10)$ & 1.00 & 3 & 65.34880 & 80.2 & 4.2 & & & & \\
\hline \multirow[t]{3}{*}{23268} & $\mathrm{~A} 0$ & & $5(16)$ & 0.69 & 1 & 55.42518 & 3.8 & 3.6 & 4.1 & 0.6 & $-20.0 \pm 8.5$ & Duflot et al. (1995) \\
\hline & & & $5(16)$ & 0.69 & 2 & 55.45426 & 3.7 & 3.6 & & & & \\
\hline & & & $5(16)$ & 0.69 & 3 & 55.48318 & 4.8 & 3.6 & & & & \\
\hline
\end{tabular}

this line, so as to mimic an increasing rotation velocity, with a Gaussian with increasing full-width at half maximum. We then cross correlated the broadened line with the original line and found no significant velocity shifts (compared to the expected repeatability error of $\overline{\sigma_{\mathrm{r}}}=2.1 \mathrm{~km} \mathrm{~s}^{-1}$ ), even for a broadening parameter which corresponded to a rotation velocity exceeding the break-up velocity. We repeated this exercise for HD 35708 (B2.5IV; $v \sin i=10-24 \mathrm{~km} \mathrm{~s}^{-1}$ ) with similar results. Although this experiment is simplistic (it neglects, e.g., noise and effects due to changes in the limb darkening), we conclude that rota- tional mismatch is not relevant at the level of $1-2 \mathrm{~km} \mathrm{~s}^{-1}$ (see also Verschueren \& David 1999). Hence, our "bias" in preferentially selecting slowly rotating standards (Sect. 2) is not significant.

\subsection{Results}

Table 3 lists the results of applying the procedure described in Sect. 4.4 to the available target spectra. Table 3 also lists $N_{\mathrm{s}}$ and $N_{\mathrm{e}}$, the number of standard stars and the number of 
Table 3. continued.

\begin{tabular}{|c|c|c|c|c|c|c|c|c|c|c|c|c|}
\hline $\begin{array}{l}\text { HD } \\
\text { (1) }\end{array}$ & $\begin{array}{l}\mathrm{SpT} \\
(2)\end{array}$ & $\begin{array}{l}\text { Mult. } \\
\text { (3) }\end{array}$ & $\begin{array}{c}N_{\mathrm{s}}\left(N_{\mathrm{e}}\right) \\
\quad(4)\end{array}$ & $\begin{array}{c}\bar{d} \\
(5)\end{array}$ & $\begin{array}{l}\text { Expos. } \\
(6)\end{array}$ & $\begin{array}{l}\text { HJD } \\
(7)\end{array}$ & $\begin{array}{l}v_{\mathrm{rad}} \\
(8)\end{array}$ & $\begin{array}{c}\sigma_{v, \mathrm{rad}} \\
(9)\end{array}$ & $\begin{array}{l}\bar{v}_{\mathrm{rad}} \\
(10)\end{array}$ & $\begin{array}{c}\sigma_{\bar{v}, \mathrm{rad}} \\
(11)\end{array}$ & $\begin{array}{c}v_{\text {lit }} \\
(12)\end{array}$ & $\begin{array}{l}\text { Remark } \\
\text { (13) }\end{array}$ \\
\hline 23478 & B3IV... & & $4(8)$ & 0.79 & 1 & 65.39109 & 15.8 & 4.0 & 15.8 & 4.0 & $25.1 \pm 3.0$ & Z83; BvA: $16.4 \pm 1.1$ \\
\hline \multirow[t]{3}{*}{23597} & B8 & & $4(9)$ & 0.67 & 1 & 65.55700 & 17.0 & 4.9 & 16.0 & 2.6 & & \\
\hline & & & $4(9)$ & 0.67 & $2^{*}$ & 65.58543 & 13.0 & 4.9 & & & & \\
\hline & & & $4(9)$ & 0.67 & 3 & 65.61373 & 17.9 & 4.8 & & & & \\
\hline 23625 & $\mathrm{~B} 2.5 \mathrm{~V}$ & $\mathrm{C}+\mathrm{SB}$ & $5(10)$ & 0.76 & 1 & 62.40834 & 1.7 & 3.0 & - & - & $20.0 \pm 1.0$ & Blaauw \& van Hoof (1963) \\
\hline \multirow[t]{3}{*}{23802} & $\mathrm{~B} 5 \mathrm{Vn}$ & $\mathrm{C}$ & $1(2)$ & 0.00 & 1 & 54.35920 & -53.8 & 1.8 & -52.3 & 6.2 & & SpT from Guetter (1977) \\
\hline & & & $1(2)$ & 0.00 & 2 & 54.38800 & -57.6 & 2.6 & & & & \\
\hline & & & $1(2)$ & 0.00 & 3 & 54.41664 & -45.5 & 0.1 & & & & \\
\hline \multirow[t]{2}{*}{24012} & B5 & & $2(3)$ & 0.37 & 1 & 54.44633 & 26.4 & 1.0 & 26.8 & 0.5 & $36.9 \pm 1.7$ & BvA; SpT from BvA \\
\hline & & & $2(3)$ & 0.37 & 2 & 54.47538 & 27.1 & 1.1 & & & & \\
\hline \multirow[t]{2}{*}{24131} & B1V & & $4(10)$ & 0.86 & $1^{*}$ & 63.57544 & 26.6 & 3.8 & 25.8 & 1.2 & $23.2 \pm 2.7$ & $\mathrm{Z} 83$ \\
\hline & & & $4(10)$ & 0.86 & $2^{*}$ & 63.59810 & 24.9 & 3.6 & & & & \\
\hline \multirow[t]{2}{*}{24190} & $\mathrm{~B} 2 \mathrm{~V}$ & SB & $5(10)$ & 0.55 & 1 & 62.56051 & 21.3 & 4.6 & - & - & $26.7 \pm 5.8$ & Lucy \& Sweeney (1971) \\
\hline & & & $5(10)$ & 0.55 & $2 *$ & 62.58902 & 22.2 & 4.6 & & & & \\
\hline 24398 & $\mathrm{~B} 1 \mathrm{Ib}$ & & 1( 3$)$ & 1.12 & 1 & 62.37038 & 20.1 & 1.2 & 20.1 & 1.2 & $21.6 \pm 4.1$ & Z83 \\
\hline \multirow[t]{5}{*}{24583} & B7V & & $4(7)$ & 1.06 & 1 & 55.55760 & 25.7 & 4.7 & 26.2 & 5.7 & $25.4 \pm 1.4$ & BvA; SpT from Guetter (1977) \\
\hline & & & $4(7)$ & 1.06 & 2 & 55.58646 & 26.7 & 4.8 & & & & \\
\hline & & & $4(7)$ & 1.06 & 3 & 55.61537 & 19.9 & 4.5 & & & & \\
\hline & & & $4(7)$ & 1.06 & 4 & 55.64456 & 35.2 & 4.8 & & & & \\
\hline & & & $4(7)$ & 1.06 & 5 & 55.67345 & 23.6 & 5.0 & & & & \\
\hline 24640 & $\mathrm{~B} 1.5 \mathrm{~V}$ & $S$ & $5(12)$ & 0.87 & 1 & 63.45678 & 22.9 & 4.1 & 22.9 & 4.1 & $17.7 \pm 0.7$ & BvA \\
\hline \multirow[t]{3}{*}{24970} & A0 & & $5(16)$ & 0.69 & $1^{*}$ & 55.33716 & 25.3 & 3.3 & 23.2 & 2.2 & $20.4 \pm 0.3$ & $\mathrm{Z} 83$ \\
\hline & & & $5(16)$ & 0.69 & 2 & 55.36676 & 23.2 & 3.5 & & & & \\
\hline & & & $5(16)$ & 0.69 & 3 & 55.39538 & 21.0 & 3.5 & & & & \\
\hline 25539 & B3V & & $4(8)$ & 0.89 & 1 & 62.43707 & 19.0 & 3.6 & 19.0 & 3.6 & $23.8 \pm 0.3$ & $\mathrm{Z} 83$ \\
\hline \multirow[t]{2}{*}{25799} & B3V... & SB & $4(8)$ & 0.89 & 1 & 62.48414 & 38.3 & 4.0 & - & - & $24.3 \pm 0.8$ & Morris et al. (1988) \\
\hline & & & $4(8)$ & 0.89 & 2 & 62.51369 & 38.4 & 3.9 & & & & \\
\hline 25833 & B5V:p & $\mathrm{C}+\mathrm{SB}$ & $2(3)$ & 0.37 & $1^{*}$ & 63.53867 & 31.6 & 1.2 & - & - & $24.7 \pm 0.9$ & Popper (1974) \\
\hline \multirow[t]{2}{*}{26499} & B9 & & $6(16)$ & 0.69 & 1 & 54.57089 & 21.8 & 3.6 & 20.3 & 2.2 & & \\
\hline & & & $6(16)$ & 0.69 & 2 & 54.60061 & 18.7 & 3.6 & & & & \\
\hline \multirow[t]{2}{*}{278942} & B3III & $\mathrm{C}$ & $2(3)$ & 0.37 & 1 & 66.43613 & 30.8 & 2.5 & 31.4 & 0.8 & & SpT from Cernis (1993) \\
\hline & & & $2(3)$ & 0.37 & 2 & 66.46811 & 32.0 & 2.8 & & & & \\
\hline \multirow[t]{2}{*}{281159} & B5V & $\mathrm{C}$ & 2(3) & 0.37 & 1 & 65.49889 & 9.1 & 1.8 & 8.5 & 0.9 & & $v_{\text {rad }}$ variable \\
\hline & & & $2(3)$ & 0.37 & 2 & 65.52757 & 7.8 & 2.1 & & & & \\
\hline
\end{tabular}

standard star exposures used in determining $v_{\mathrm{rad}}$, respectively, and the mean radial velocity for each exposure (Eq. (3)); the corresponding standard deviation is denoted $\sigma_{v, \text { rad }}$. The mean distance in luminosity class-spectral type space (Fig. 5) between the target and the $N_{\mathrm{e}}$ standard star exposures is denoted $\bar{d} \equiv N_{\mathrm{e}}^{-1} \cdot \sum_{i=1}^{N_{\mathrm{e}}} d_{i}$. In general, the effect of template mismatch increases with increasing $\bar{d}$. Table 3 also lists, for each target star, the time-averaged radial velocity $\bar{v}_{\text {rad }}$, and the corresponding standard deviation $\sigma_{\bar{v} \text {,rad }}$, of the $N$ radial velocities corresponding to the $N$ exposures obtained for each star. The quantity $\bar{v}_{\text {rad }}$ is the best estimate of the true radial velocity of an object, provided it is single; $\bar{v}_{\text {rad }}$ is potentially less straightforward to interpret otherwise.

Table 3 shows that, in general, $\sigma_{v, \text { rad }} \gg \sigma_{\bar{v} \text {,rad }} \gtrsim \overline{\sigma_{\mathrm{r}}}$. The first inequality suggests that template mismatch, combined with possible errors in the assumed radial velocities for the standard stars, is a significant error source, justifying our averaging-approach. The similar magnitudes of $\sigma_{\bar{v} \text {,rad }}$ (with a mean value for all stars of $\left.1.5 \mathrm{~km} \mathrm{~s}^{-1}\right)$ and the mean repeatability error $\left(\overline{\sigma_{\mathrm{r}}}=2.1 \mathrm{~km} \mathrm{~s}^{-1}\right)$ provide a further indication of the validity of our approach.

The errors $\sigma_{\bar{v} \text {,rad }}$ on $\bar{v}_{\text {rad }}$ are sometimes remarkably small, down to a few tenths of a $\mathrm{km} \mathrm{s}^{-1}$. Given that template mismatch can have an effect at the level of $\sim 3.0-5.0 \mathrm{~km} \mathrm{~s}^{-1}$ (corresponding to $\sigma_{v, \text { rad }}$ ), that the mean repeatability error is $2.1 \mathrm{~km} \mathrm{~s}^{-1}$ (Sect. 4.3), and that the expected error due to uncertainties in the radial velocities of the standard stars is also $\sim 2.0 \mathrm{~km} \mathrm{~s}^{-1}$ (Sect. 4.1), we suspect that increasing $\sigma_{\bar{v} \text {,rad }}$ to $2.0 \mathrm{~km} \mathrm{~s}^{-1}$ provides a more realistic estimate of the error.

The 11 target exposures identified in Sect. 3 as suspect as a result of detector instability are indicated with asterisks in Col. 6 of Table 3. Comparing the associated radial velocities 
with non-suspect exposures shows no significant deviations, consistent with the relatively small magnitude of the effect established in Sect. $3\left(0.6-2.9 \mathrm{~km} \mathrm{~s}^{-1}\right.$; Fig. 2). We therefore do not discriminate these suspect exposures further and treat them as normal in the remainder of this manuscript. They thus also contribute to the time-averaged radial velocities $\bar{v}_{\text {rad }}$.

Table 4 shows, as a representative example, how the final, mean radial velocities $v_{\text {rad }}$ in Table 3 (Eq. (3)) are built up for the A0 star HD 24970. This star was observed $N=3$ times and has $N_{\mathrm{s}}=5$ suitable standard stars (Fig. 5), giving rise to $N_{\mathrm{s}}=5$ radial velocities per exposure. The best-match radial velocity standard (HD 196724, A0V, $d=0.00$, and $w=1.00$ ) implies the target star's radial velocity is $\sim 26 \mathrm{~km} \mathrm{~s}^{-1}$. The other four standards, however, which have rather closely matching spectral classifications, imply a velocity of $\sim 20-25 \mathrm{~km} \mathrm{~s}^{-1}$. Two explanations, which are not mutually exclusive, may be invoked to reconcile this discrepancy: either template mismatch explains the systematically lower values, or an error in the literature radial velocity of the A0V standard HD 196724 explains the systematically higher value. Discriminating between these two options is impossible using the available data. The problem is, however, automatically "solved" for/alleviated in the weighted-average approach adopted here.

Table 5 shows, for the single A0 stars HD $24970(N=3$; $\left.\sigma_{\mathrm{r}}=2.7 \mathrm{~km} \mathrm{~s}^{-1}\right)$ and $23268\left(N=3 ; \sigma_{\mathrm{r}}=1.4 \mathrm{~km} \mathrm{~s}^{-1}\right)$, for all combinations of the $N=3$ object exposures and the $N_{\mathrm{s}}=5$ suitable standard stars, the standard deviations of the radial velocities corresponding to the different exposures of the standard stars (cf. Table 4). The standard deviations are, in some cases, slightly larger than the expected values (roughly the repeatability errors), suggesting that both errors in the literature radial velocities of standard stars and template mismatch contribute significantly to the total error budget.

\subsection{Spectroscopic binaries}

Our sample contains five known spectroscopic binaries (SBs; a sixth SB, X Per/HD 24534, was classified as Per OB2 member by B52, but was not observed by us; see Sect. 5.2.1). Three of these have previously been identified as double-lined SBs (SB2s). The modest resolving power of the spectrographgrating combination used by us $(R \sim 7000)$, combined with the very broad hydrogen absorption lines in the spectra of these SB2s, do not allow a proper decomposition of the spectra in all of these cases (we did not optimize our instrumental setup for SB2s). We were therefore forced to treat these spectra as arising from a single star. The resulting radial velocities, derived by means of cross correlation using a template star matched to the spectral type and luminosity class of the primary component, are of modest physical significance. Conceptually, they correspond to a luminosity-weighted average of the instantaneous radial velocities of the primary and secondary components, with template mismatch complicating matters (recall that the standard star was selected based on the primary component exclusively). While the composite spectrum we observed is a luminosity-weighted average of the instantaneous Dopplershifted spectra of the two components, the inferred radial velocity is not necessarily a luminosity-weighted average of the instantaneous radial velocities of the components, although a trend along these lines might be expected.

The orbital elements from the literature for the five known SBs in our sample are listed in Table 6. We have one or a few measurements for each of these objects. Repeat measurements were usually taken on the same night, typically within one hour, with the exception of o Per (Table 3). As shown in Table 6, the amplitudes of the radial velocity variations are large, ranging up to $\sim 180 \mathrm{~km} \mathrm{~s}^{-1}$ for AG Per. With the caveat mentioned above for SB2s, our instantaneous measurements fall within these ranges in all five cases.

For the two single-lined SBs (the SB1s), we used the known periods to investigate whether (i) our repeat measurements should have shown significant differences, and (ii) whether we could reconstruct what the expected $v_{\text {rad }}$ should have been at the epoch of our observations. As the periods are known to sufficient accuracy, it is possible to do this, and we find agreement in both cases. We discuss each binary in more detail below.

HD 23180 (o Per): this well-known SB2 (e.g., Stickland \& Lloyd 1998) was observed twice (the first exposure being suspect), and then once more, $\sim 1.75$ days later. According to Hipparcos, o Per is also an $11^{\prime \prime} 019$ visual binary with $\Delta H p=$ 2.91 mag (Table 1; see Stickland \& Lloyd for a discussion of this component). The Hipparcos data imply that our observations have primarily ( $93 \%$ in flux) detected photons from the B1III + B2V SB. A simple-minded comparison of our radial velocities, obtained by simply ignoring the presence of the secondary component, with predictions based on Stickland \& Lloyd's (1998) orbital ephemeris shows the expected "fluxweighted-average" trend (Table 6). The third exposure of o Per shows some double helium lines with an average velocity separation of $\sim 200 \pm 30 \mathrm{~km} \mathrm{~s}^{-1}$; this is consistent with the expected velocity separation for this exposure of $\sim 230 \mathrm{~km} \mathrm{~s}^{-1}$ (Table 6).

HD 23625: this object was studied by Blaauw \& van Hoof (1963), who classified it as a B2V SB2. HD 23625 is also a Hipparcos component binary with $\Delta H p=2.58 \mathrm{mag}$ and $\rho=3$.'349 (Table 1); these data imply that this fainter component cannot have been present in the $3^{\prime \prime}$-diameter entrance pupil of the image slicer. We observed this object once, and find $v_{\text {rad }}=1.7 \pm 3.0 \mathrm{~km} \mathrm{~s}^{-1}$ (our spectrum superficially looks single-lined, although a slight asymmetry in the line profiles is present; the radial velocity was again obtained by ignoring the presence of the secondary component in the spectrum). According to Blaauw \& van Hoof's ephemeris, we should have obtained $-34.7 \pm 19.1 \mathrm{~km} \mathrm{~s}^{-1}$ for the primary component. This prediction agrees with our measurement at the $2 \sigma$-level.

HD 24190: this object was identified as a low-amplitude B2V SB1 by Blaauw \& van Albada (1963; BvA). According to the improved ephemeris from Lucy \& Sweeney (1971), our exposures $\left(21.3 \pm 4.6\right.$ and $\left.22.2 \pm 4.6 \mathrm{~km} \mathrm{~s}^{-1}\right)$ should have read $20.6 \pm 1.9$ and $20.5 \pm 1.9 \mathrm{~km} \mathrm{~s}^{-1}$, respectively. We conclude our measurements are in full agreement with Lucy \& Sweeney's ephemeris.

HD 25799: this object was classified as a low-amplitude eclipsing B3V SB1 with a poorly determined/variable period by BvA $\left(P \sim 10.67 \mathrm{~d}\right.$ and $\left.K_{1}=20 \mathrm{~km} \mathrm{~s}^{-1}\right)$. BvA's discovery of "erratic 
Table 4. Illustration of results underlying the mean radial velocities $v_{\text {rad }}$ in Table 3 (cf. Eq. (3)). The star HD 24970 (A0) was observed $N=3$ times and has $N_{\mathrm{s}}=5$ suitable standard stars, giving rise to 5 radial velocities per exposure. These values are listed (in km s${ }^{-1}$ ), together with the spectral classifications and distances $d$ and weights $w$ (see Eqs. (1) and (4)) of the standard stars. The distance-weighted mean radial velocities with their errors are listed in the final column (in $\mathrm{km} \mathrm{s}^{-1}$ ). Systematic differences between columns in this table can be due to template mismatch and/or incorrect literature radial velocities; in general, systematic differences between lines in this table can be due to random errors (statistics), instrumental shifts, and/or multiplicity (not likely in this specific case).

\begin{tabular}{rcccccc}
\hline \hline HD & 10982 & 196724 & 196821 & 27638 & 58142 & $v_{\text {rad }}$ \\
SpT & B9V & A0V & A0III & B9V & A1V & \\
$d=$ & 1.00 & 0.00 & 1.00 & 1.00 & 1.00 & \\
$w=$ & 0.50 & 1.00 & 0.50 & 0.50 & 0.50 & \\
\hline Exposure $1^{*}$ & 26.2 & 27.8 & 24.3 & 21.4 & 22.8 & $25.3 \pm 3.3$ \\
Exposure 2 & 24.1 & 26.1 & 21.6 & 20.1 & 20.2 & $23.2 \pm 3.5$ \\
Exposure 3 & 21.6 & 23.8 & 19.6 & 16.9 & 18.4 & $21.0 \pm 3.5$ \\
\hline
\end{tabular}

Table 5. The standard deviations of the radial velocities corresponding to the $N_{\mathrm{e}}$ different exposures of the $N_{\mathrm{s}}=5$ suitable standard stars for all combinations of the different object exposures and suitable standard stars of the stars HD 24970 (A0; cf. Table 4; top part) and HD 23268 (A0; bottom part). The distance-weighted mean radial velocities with their errors are listed in the final column (in $\mathrm{km} \mathrm{s}^{-1}$ ); time-averaged values, denoted $\bar{v}_{\text {rad }}$, are provided in Table 3. The quantity $\sigma_{\mathrm{r}}$ denotes the repeatability error (Sect. 4.3).

\begin{tabular}{rcccccc}
\hline \hline HD & 10982 & 196724 & 196821 & 27638 & 58142 & $v_{\text {rad }}$ \\
SpT & B9V & A0V & A0III & B9V & A1V & \\
$d=$ & 1.00 & 0.00 & 1.00 & 1.00 & 1.00 & \\
$w=$ & 0.50 & 1.00 & 0.50 & 0.50 & 0.50 & \\
$N=$ & 2 & 5 & 3 & 2 & 4 & \\
$\sigma_{\mathrm{r}}=$ & 3.4 & 2.3 & 0.9 & 0.2 & 2.0 & \\
\hline Exposure 1* & 1.8 & 3.4 & 0.4 & 0.2 & 2.7 & $+25.3 \pm 3.3$ \\
Exposure 2 & 2.3 & 3.5 & 0.9 & 0.6 & 2.7 & $+23.2 \pm 3.5$ \\
Exposure 3 & 2.3 & 3.5 & 0.9 & 0.7 & 2.6 & $+21.0 \pm 3.5$ \\
\hline Exposure 1 & 2.1 & 3.5 & 0.8 & 0.1 & 2.6 & $+3.8 \pm 3.6$ \\
Exposure 2 & 2.2 & 3.5 & 1.0 & 0.6 & 2.6 & $+3.7 \pm 3.6$ \\
Exposure 3 & 2.3 & 3.6 & 1.0 & 0.6 & 2.6 & $+4.8 \pm 3.6$ \\
\hline
\end{tabular}

velocity changes" was in fact the first indication that this object is not a normal binary. Morris et al. (1988) showed that an improved ephemeris (notably $P=0.9121679 \mathrm{~d}$ ) could fit all previous spectroscopic data, but also showed, based on new photometric data, that the object is not a binary but a nonradial pulsator. This finding was confirmed later by Hipparcos photometry. We observed this object twice, obtaining $v_{\text {rad }}=$ $38.3 \pm 4.0 \mathrm{~km} \mathrm{~s}^{-1}$ and $38.4 \pm 3.9 \mathrm{~km} \mathrm{~s}^{-1}$. These observations are consistent with Morris et al.'s ephemeris within $1 \sigma$, which predicts $v_{\mathrm{rad}}=40.3 \pm 2.1$ and $41.2 \pm 1.7 \mathrm{~km} \mathrm{~s}^{-1}$, respectively.

HD 25833 (AG Per): this object is one of the few detached massive eclipsing SB2s, and is very well-studied (e.g., Popper 1974; cf. Popper \& Hill 1991). The system is eccentric ( $e=$ $0.071 \pm 0.001)$ and shows apsidal motion with a period of $75.6 \pm 0.6 \mathrm{yr}$ (i.e., $4.76^{\circ} \mathrm{yr}^{-1}$ ). Spectroscopic ephemeris predictions are non trivial as a result. As both components are roughly equally bright $(V \sim 7.46$ and $\sim 7.88 \mathrm{mag}$; Table 6 in Giménez \& Clausen 1994) and have similar spectral types (B4V + B5; Giménez \& Clausen), we expect the radial velocity inferred from our low-resolution composite spectrum to be near the systemic velocity, with a relatively small "flux-weightedaverage" oscillation due to orbital motion. Additional complications might arise from the presence of a third component, with Hipparcos parameters $\Delta H p=1.81 \mathrm{mag}$ and $\rho=0{ }^{\prime} 803$
(Table 1). Giménez \& Clausen (1994) provide the most recent photometric eclipse ephemeris, from which we derive that the single, formally suspect, exposure we took of this object should have an associated phase of $\sim 0.20$ with respect to a secondary eclipse. This roughly implies radial velocities for the primary of $\gamma+K_{1} \sim+24.7+162.8 \sim+190 \mathrm{~km} \mathrm{~s}^{-1}$ and for the secondary of $\gamma-K_{2} \sim+24.7-178.7 \sim-150 \mathrm{~km} \mathrm{~s}^{-1}$, so that the expected velocity separation is $\sim 340 \mathrm{~km} \mathrm{~s}^{-1}$. Not surprisingly, the hydrogen lines in our composite spectrum do not show clear double-lined signs (although an asymmetry of the line profiles seems present), but the much narrower helium lines are double. From these, we derive a mean velocity separation of $329 \pm 17 \mathrm{~km} \mathrm{~s}^{-1}$ (based on 5 double lines), consistent with expectations. Ignoring the presence of the secondary component in the composite spectrum, we derive $v_{\text {rad }}=31.6 \pm 1.2 \mathrm{~km} \mathrm{~s}^{-1}$ (based mainly on the broad hydrogen lines in the spectrum), which is indeed close to the systemic velocity.

\subsection{Comments on individual stars}

We noted in Sect. 4.4 that five targets have Hipparcos spectral types and luminosity classes which differ significantly from other literature sources. In all these cases, we dropped the Hipparcos data and adopted the classifications from the 
Table 6. The five spectroscopic binaries (SBs) and their orbital elements. Columns for the first part of the table (literature data): (1) HD number; (2) periastron date $T_{0}(\mathrm{HJD})$; (3) period $P$ (days); (4) primary orbital semi-amplitude $K_{1}\left(\mathrm{~km} \mathrm{~s}^{-1}\right)$; (5) secondary orbital semi-amplitude $K_{2}$ $\left(\mathrm{km} \mathrm{s}^{-1}\right.$; only for double-lined spectroscopic binaries, i.e., SB2s); (6) systemic velocity $\gamma\left(\mathrm{km} \mathrm{s}^{-1}\right)$; (7) orbital eccentricity $\epsilon$ (see the footnote to the table for literature sources). Columns for the second part of the table (data from this study): (1) HD number; (2) exposure number (Table 3); (3) instantaneous radial velocity $\left(\mathrm{km} \mathrm{s}^{-1}\right)$; (4) primary component prediction using the ephemeris provided above $\left(\mathrm{km} \mathrm{s}^{-1}\right)$; (5) secondary component prediction using the ephemeris provided above ( $\mathrm{km} \mathrm{s}^{-1}$; only for SB2s).

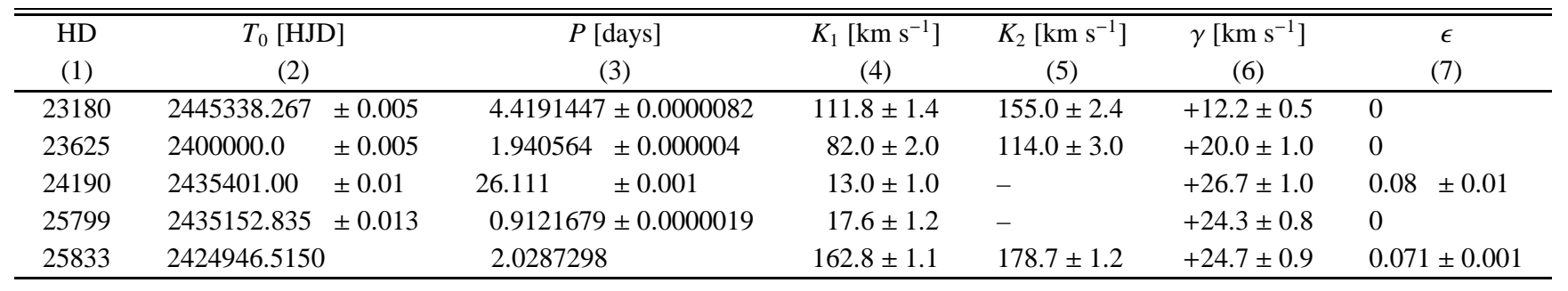

HD 23180: Stickland \& Lloyd (1998);

HD 23625: Blaauw \& van Hoof (1963);

HD 24190: Lucy \& Sweeney (1971);

HD 25799: Morris et al. (1988); this is a non-radial pulsator;

HD 25833: Giménez \& Clausen (1994) (cf. Popper 1974).

\begin{tabular}{ccrrrr}
\hline \hline HD & Exposure & $v_{\text {rad }}\left[\mathrm{km} \mathrm{s}^{-1}\right]$ & \multicolumn{1}{c}{$v_{\text {rad }, 1}\left[\mathrm{~km} \mathrm{~s}^{-1}\right]$} & \multicolumn{2}{c}{$v_{\text {rad, }, 2}\left[\mathrm{~km} \mathrm{~s}^{-1}\right]$} \\
$(1)$ & $(2)$ & \multicolumn{1}{c}{$(3)$} & \multicolumn{1}{c}{$(4)$} & \multicolumn{1}{c}{$(5)$} & \\
\hline 23180 & $1^{*}$ & $-6.1 \pm 3.3$ & $-28.0 \pm 1.8$ & $67.9 \pm 2.5$ \\
& 2 & $6.4 \pm 3.1$ & $-21.6 \pm 1.8$ & $59.0 \pm 2.5$ \\
& 3 & $80.2 \pm 4.2$ & $108.7 \pm 1.6$ & $-121.6 \pm 2.5$ \\
23625 & 1 & $1.7 \pm 3.0$ & $-34.7 \pm 19.1$ & $96.1 \pm 26.5$ \\
24190 & 1 & $21.3 \pm 4.6$ & $20.6 \pm 1.9$ & & - \\
& $2 *$ & $22.2 \pm 4.6$ & $20.5 \pm 1.9$ & & - \\
25799 & 1 & $38.3 \pm 4.0$ & $40.3 \pm 2.1$ & & - \\
& 2 & $38.4 \pm 3.9$ & $41.2 \pm 1.7$ & & - \\
25833 & $1 *$ & $31.6 \pm 1.2$ & $\sim 190$ & $\sim-150$ & \\
\hline
\end{tabular}

dedicated studies. The implications of this choice for the inferred radial velocities are discussed below.

HD 20987: the Hipparcos spectral type (B9) suggests $v_{\text {rad }} \sim$ $-40 \mathrm{~km} \mathrm{~s}^{-1}$. Abt (1985) and Roman (1978), independently, quote a spectral classification of $\mathrm{B} 2 \mathrm{~V}$, which suggests $v_{\text {rad }}=$ $-22.1 \pm 0.8 \mathrm{~km} \mathrm{~s}^{-1}$ (Table 3; we used $d_{\max }=0.75$ ).

HD 23802: the Hipparcos spectral type (B9) suggests $v_{\text {rad }} \sim$ -70 to $-80 \mathrm{~km} \mathrm{~s}^{-1}$. Guetter (1977) quotes a spectral classification of B5Vn, which results in $v_{\mathrm{rad}}=-52.3 \pm 6.2 \mathrm{~km} \mathrm{~s}^{-1}$ (Table 3; we used $d_{\max }=0.75$ ). The three exposures give a relatively large spread in the inferred radial velocities $\left(\sim 12 \mathrm{~km} \mathrm{~s}^{-1}\right)$, which, if significant, suggests that the radial velocity of this object is variable, most likely as a result of duplicity.

HD 24012: the Hipparcos spectral type (B9) suggests $v_{\text {rad }} \sim$ $19 \mathrm{~km} \mathrm{~s}^{-1}$. BvA quote a spectral classification of B5, which suggests $v_{\text {rad }}=26.8 \pm 0.5 \mathrm{~km} \mathrm{~s}^{-1}$ (Table 3 ).

HD 24583: the Hipparcos spectral type (A0) suggests $v_{\text {rad }} \sim$ $22 \mathrm{~km} \mathrm{~s}^{-1}$. Guetter (1977) quotes a spectral classification of B7V, which suggests $v_{\text {rad }}=26.2 \pm 5.7 \mathrm{~km} \mathrm{~s}^{-1}$ (Table 3). Our five exposures seem to contain 1-2 outliers (exposure 4 and, to a smaller extent, exposure 3). Excluding exposure 4 returns $\bar{v}_{\text {rad }}=24.0 \pm 3.0 \mathrm{~km} \mathrm{~s}^{-1}$; excluding both exposures returns $\bar{v}_{\text {rad }}=25.4 \pm 1.6 \mathrm{~km} \mathrm{~s}^{-1}$.
HD 278942: this faint object is a Hipparcos component binary $(\rho=0.149$ and $\Delta H p=0.78 \mathrm{mag}$; Table 1$)$. An IRAS ring in the interstellar medium around this star explains the various colour and spectral type measurements reported in the literature (see Z99 for details; Hipparcos/Tycho: SpT = F2 and $B-V=1.13 \mathrm{mag}$; SIMBAD/AGK3: SpT = B5 and $B-V=-0.1 \mathrm{mag}$; Cernis 1993: SpT = B3III; Andersson et al. 2000: SpT = O9.5V-B0V; the latter authors suggested that the IRAS ring, which is also visible at radio wavelengths, is an HII region associated with the object). Our spectra, although noisy, show that the object is a B-type rather than an F-type star. We therefore adopt the B3III spectral type from Cernis (1993), who identified HD 278942 as a "possible photometric B3III + F5I binary". This choice implies a radial velocity of $\sim 31 \mathrm{~km} \mathrm{~s}^{-1}$ (Table 3; despite a repeatability error of $\sim 16 \mathrm{~km} \mathrm{~s}^{-1}$, the inferred radial velocities of the two exposures are consistent at the level of $\sim 1 \mathrm{~km} \mathrm{~s}^{-1}$ ). Using the SIMBAD/AGK3 B5 spectral type would result in a radial velocity of $\sim 38 \mathrm{~km} \mathrm{~s}^{-1}$; Andersson's classification would imply a radial velocity of $\sim 70-90 \mathrm{~km} \mathrm{~s}^{-1}$.

HD 281159: we observed this star twice (in subsequent exposures), obtaining $v_{\text {rad }}=9.1 \pm 1.8$ and $7.8 \pm 2.1 \mathrm{~km} \mathrm{~s}^{-1}$. Both SIMBAD and Duflot et al. (1995; the WEB catalogue of radial velocities) list a literature radial velocity of $+14 \pm 5 \mathrm{~km} \mathrm{~s}^{-1}$, 
which seems in reasonable agreement at first sight. There is, however, a long history behind these values. B52, based on Moore's (1932) results, lists a radial velocity of $+32 \mathrm{~km} \mathrm{~s}^{-1}$ and sets a radial velocity variability flag. Wilson \& Joy (1952) list " $+25 \mathrm{~km} \mathrm{~s}^{-1}$ (variable radial velocity)". These authors based their mean value on 4 measurements: $+42,+20,+80$, and $-43 \mathrm{~km} \mathrm{~s}^{-1}$. The General Catalogue of Radial Velocities (GCRV; 1953) lists $+25 \pm 5 \mathrm{~km} \mathrm{~s}^{-1}$ and does not mention velocity variability. Petrie \& Pearce (1961) list “ $+7.0 \pm$ $4.7 \mathrm{~km} \mathrm{~s}^{-1}$ (variable radial velocity)". These authors based their mean value on 6 measurements: $+34,-10,-3,-4,+19$, and $+16 \mathrm{~km} \mathrm{~s}^{-1}$. Evans (1967; "The revision of the GCRV") lists $+14 \pm 5 \mathrm{~km} \mathrm{~s}^{-1}$ based on 10 measurements. This mean value is the number-of-measurements-weighted average of the mean results of Wilson \& Joy (4 measurements) and Petrie \& Pearce (6 measurements). Evans did not copy any of the variability flags in the literature, and his result $\left(+14 \pm 5 \mathrm{~km} \mathrm{~s}^{-1}\right)$ erroneously suggests that the radial of HD 281159 is well-defined and reliable. Evans's results were copied by both Duflot et al. (1995) and SIMBAD. We thus conclude that the agreement between the results of the latter two literature sources with our measurements is spurious and we suspect that this object is an SB. As the radial velocity determinations by Wilson \& Joy and Petrie \& Pearce have been published without the associated epochs, we cannot analyse the data for this star in the light of an SB model. Our mean radial velocity $\left(\bar{v}_{\text {rad }}=8.4 \pm 0.9 \mathrm{~km} \mathrm{~s}^{-1}\right)$ should be treated with care.

\subsection{External accuracy check}

Our list of 29 stars with $v_{\text {rad }}$ measurements contains the five confirmed spectroscopic binaries discussed in Sect. 4.7, and we have seen that our measured values are consistent with earlier measurements. We were able to find reported radial velocity measurements for 15 of the remaining 24 objects. The main sources, in order of decreasing preference, are Zentelis (1983; Z83), Blaauw \& van Albada (1963; BvA), Blaauw (1952; B52), and Grenier et al. (1999).

Figure 6 shows our measured values $\left(v_{\mathrm{OHP}}\right)$ for these stars versus those in the literature $\left(v_{\text {lit }}\right)$. We have excluded HD 23268. Its literature radial velocity from Duflot et al. (1995) of $-20 \pm 8.5 \mathrm{~km} \mathrm{~s}^{-1}$ is based on 3 measurements with unknown source; it is consistent with our measurement of $4.1 \pm 0.6 \mathrm{~km} \mathrm{~s}^{-1}$ at the $3 \sigma$ level. There is reasonable agreement between the literature values and our measurements. A small offset from the diagonal can be expected, as we have set the zero of our scale by the choice of standards, while in literature studies this is done in various different ways (cf. Sect. 4.1). We find that the weighted mean and dispersion of $\Delta \equiv v_{\mathrm{OHP}}-v_{\text {lit }}$ are 0.5 and $3.3 \mathrm{~km} \mathrm{~s}^{-1}$ (the straight mean and dispersion of $\Delta \equiv\left(v_{\mathrm{OHP}}-v_{\mathrm{lit}}\right) / \sqrt{\sigma_{v, \mathrm{OHP}}^{2}+\sigma_{v, \text { lit }}^{2}}$ are $0.1 \pm 0.9$, compared to the expected value of $0 \pm 1$ ). These values are for the complete set of single stars plus the $2+2$ SB1 exposures for HD 24190 and 25799 (Fig. 6). We excluded HD 23268 and the three "outliers above the diagonal" (HD 23060, 23478, and 24012) from this sample. Removing the SB1 exposures from the sample results in 0.9 and $3.5 \mathrm{~km} \mathrm{~s}^{-1}$ (and $0.2 \pm 1.0$ ),

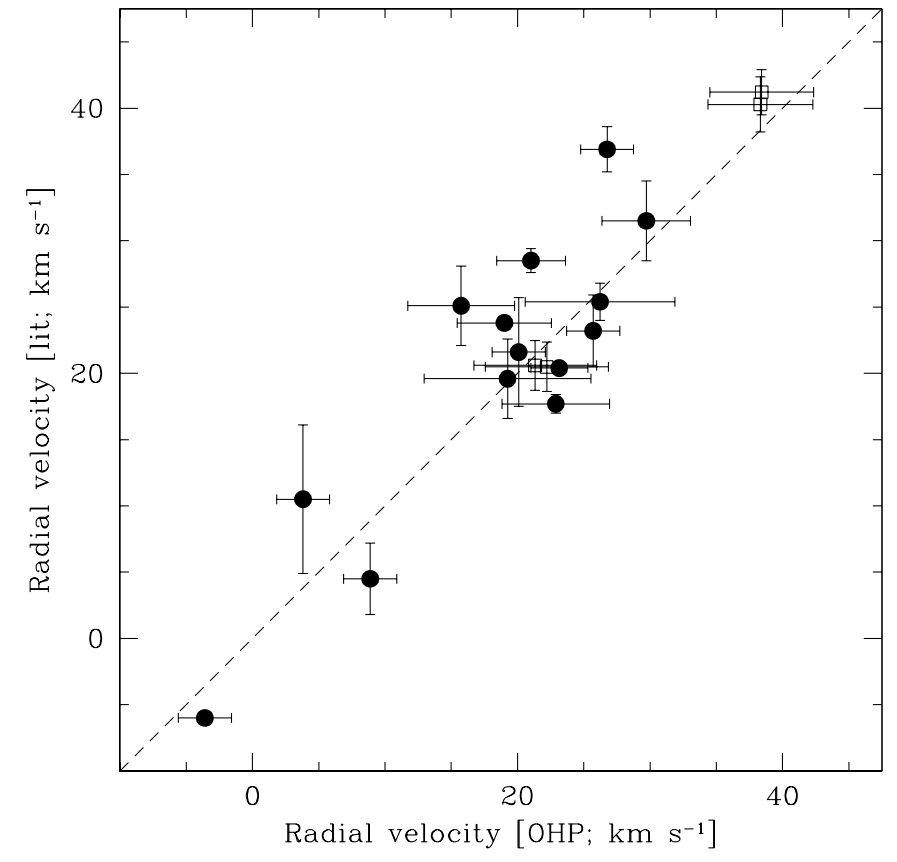

Fig. 6. Comparison of OHP and literature radial velocities. The filled circles denote 15 single stars (i.e., non-SBs). One of these, HD 23268 with OHP and literature radial velocities of $4.1 \pm 0.6$ and $-20 \pm$ $8.5 \mathrm{~km} \mathrm{~s}^{-1}$ (Duflot et al. 1995), respectively, falls outside the plot. The four open squares refer to the $2+2$ instantaneous measurements of the two single-lined SBs HD $24190\left(v_{\text {rad }} \sim 22 \mathrm{~km} \mathrm{~s}^{-1}\right)$ and 25799 $\left(v_{\text {rad }} \sim 38 \mathrm{~km} \mathrm{~s}^{-1}\right)$.

while adding the three outliers results in -1.5 and $5.4 \mathrm{~km} \mathrm{~s}^{-1}$ (and $-0.4 \pm 1.6$ ).

\section{Interpretation}

We now use our measurements, together with those for other certain and proposed members of Per OB2, to study the association. We first discuss the distribution of radial velocities (Sect. 5.1) and membership of individual stars (Sect. 5.2), briefly address the internal structure (Sect. 5.3), and then analyse the colour-magnitude diagram (Sect. 5.4).

\subsection{Distribution of radial velocities}

We use our own measurements for 24 stars, and use the reported systemic $(\gamma)$ velocities for the five spectroscopic binaries (Table 6) in our sample of candidate Per OB2 members. The results are plotted in Fig. 7, which shows the distribution of these objects on the sky, flanked by plots of $v_{\text {rad }}$ versus galactic longitude $\ell$ and latitude $b$. We use different symbols for our new measurements (squares) and the literature values (triangles).

The $v_{\text {rad }}$ versus $\ell$ plot clearly shows a clump of stars near $v_{\text {rad }} \sim 23 \mathrm{~km} \mathrm{~s}^{-1}$, containing many of the classical Per OB2 members (B52). The $v_{\text {rad }}$ versus $b$ plot shows a very similar separation. In addition to a few outliers, there is a second clump of stars, covering a larger range in $\ell$, with a small dispersion. The measured values coincide very nicely with the expected $v_{\text {rad }}$ for field stars in the direction of Per OB2, i.e., those obeying simple galactic rotation (in this direction, however, most 


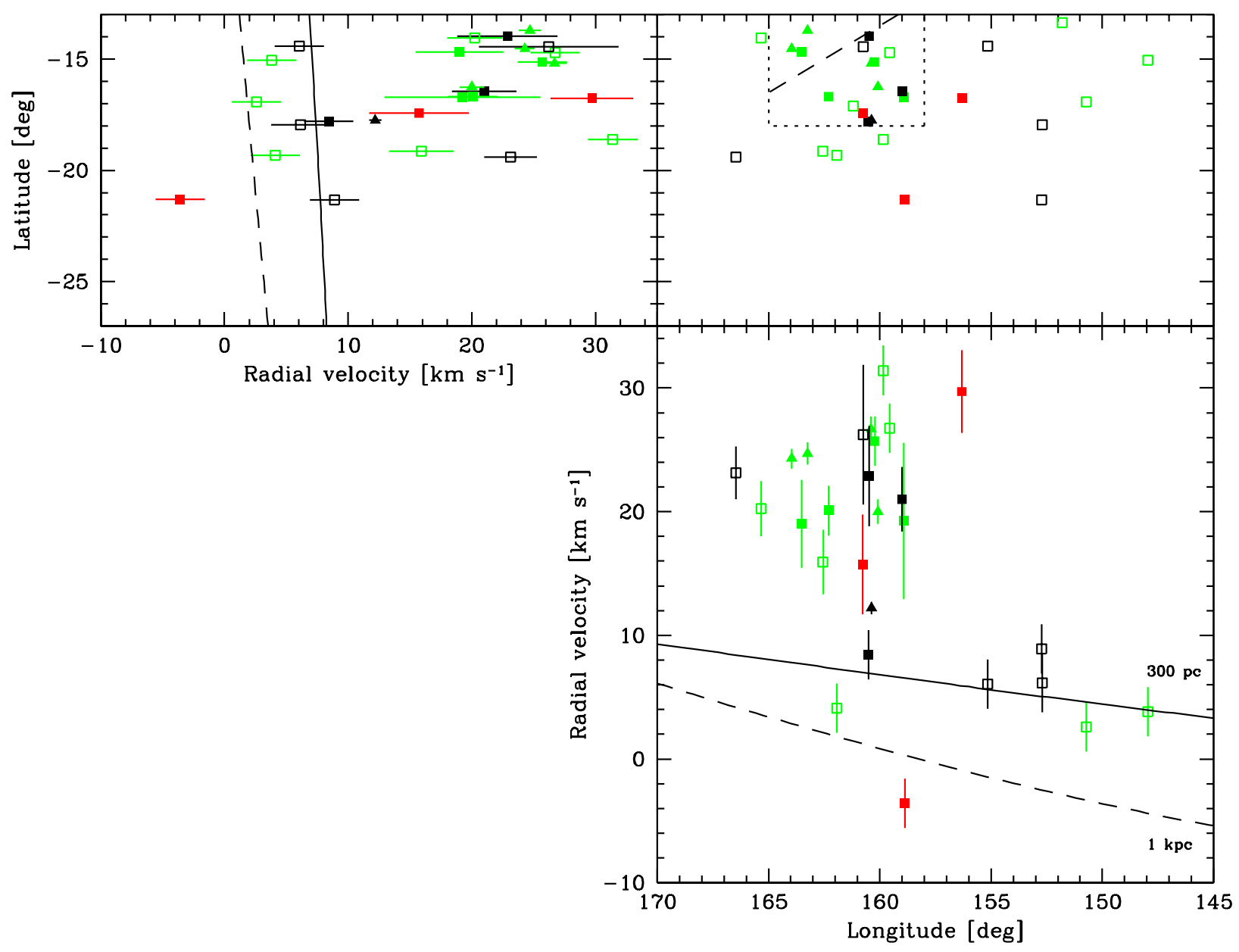

Fig. 7. The radial velocity measurements of our 29 targets as a function of position on the sky. Squares denote our own radial velocity measurements (24 stars), while triangles denote reported systemic velocities for the five spectroscopic binaries (Table 6). Open symbols refer to certain and possible Hipparcos members (Z99), while filled symbols indicate 15 of the 17 classical Per OB2 members from B52; significant overlap between these groups exists (Sect. 5.2.1). The radial velocities of HD 20987 and 23802 fall outside the radial-velocity panels (top left and bottom right). The field of view (top right panel) is that used by Z99 in their Hipparcos analysis of the Per OB2 association. The dotted box and dashed line on the sky denote Belikov et al.'s (2002b) approximate extent of Per OB2 and the separation between their alleged subgroups a and $\mathrm{b}$ (see their Fig. 5). The lines in the radial-velocity panels indicate the predicted radial velocities for disk stars at $300 \mathrm{pc}$ (full line) and $1 \mathrm{kpc}$ (dashed line).

of the observed radial velocity is reflected Solar motion). We conclude that these objects are unrelated field stars.

The radial velocity separation between the association and the Galactic disk allows us to determine the mean radial velocity of the group. Our list of 29 stars contains 19 stars with radial velocities between 10 and $35 \mathrm{~km} \mathrm{~s}^{-1}$. From this list, we reject the likely non-member o Per (HD 23180; see Sect. 5.2). Figure 8 shows the radial velocity histogram of the 18 (candidate) members. From this histogram, we obtain a mean radial velocity of $23.5 \mathrm{~km} \mathrm{~s}^{-1}$ and an associated dispersion $\sigma=3.9 \mathrm{~km} \mathrm{~s}^{-1}$. This mean velocity is consistent with the value derived by Blaauw (1944), $19.4 \pm 1.7 \mathrm{~km} \mathrm{~s}^{-1}$.

The dispersion of $3.9 \mathrm{~km} \mathrm{~s}^{-1}$ amongst the measured radial velocities in Per OB2 provides an a posteriori external check of the accuracy of our measurements, as it is an upper limit on this (the measured dispersion arises from measurement errors, internal velocity dispersion in the association, unrecognized duplicity, and/or the presence of non-members in the sample). The internal dispersion is probably only $1-3 \mathrm{~km} \mathrm{~s}^{-1}$ (Z99), which suggests that the external accuracy is $\sim 3 \mathrm{~km} \mathrm{~s}^{-1}$. This is in harmony with the repeatability errors and the analysis of Sect. 4.9.

\subsection{Membership}

The clean separation of the association and the local disk stars in radial velocity (Fig. 7) makes it possible to improve some of the earlier membership assignments (notably B52 and Z99). We discuss these in some detail here.

\subsubsection{Blaauw (1952) (B52)}

B52 identified 17 stars as member of Per OB2. Z99 confirmed eight of these: HIP 17313, 17735, 18081, 18111, 18246, 19039, 19178, and 19201 (HD 22951, 23625, 24131, 24190, $24398,25539,25799$, and 25833). Four of these eight are SBs (Table 6); their systemic radial velocities (from the literature) 


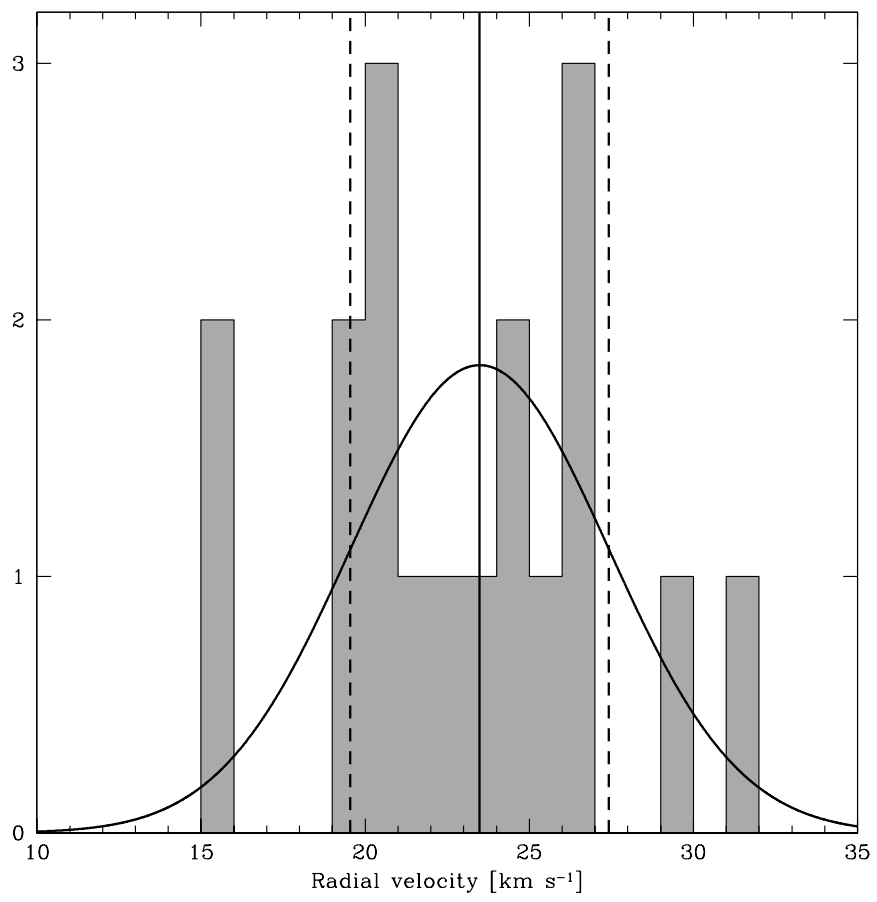

Fig. 8. Radial velocity histogram of all 18 stars with OHP radial velocities between 10 and $35 \mathrm{~km} \mathrm{~s}^{-1}$ (we excluded o Per/HD 23180; Sect. 5.2). We find a mean radial velocity of $23.5 \mathrm{~km} \mathrm{~s}^{-1}$ (vertical line) and an associated dispersion of $3.9 \mathrm{~km} \mathrm{~s}^{-1}$ (dashed vertical lines). The curve denotes a reference Gaussian with a mean of $23.5 \mathrm{~km} \mathrm{~s}^{-1}$ and $\sigma=3.9 \mathrm{~km} \mathrm{~s}^{-1}$. See Sect. 5.1 for details.

cluster around $20-25 \mathrm{~km} \mathrm{~s}^{-1}$. The OHP radial velocities of the other four objects cluster in the same range. We thus conclude that all eight B52/Z99 Per OB2 members are genuine members.

Z99 identified four B52 members as possible astrometric member: HIP 17387, 17448, 17465, and 18434 (HD 23060, 23180,281159 , and 24640). We observed all of them, and conclude that HD 23180 (o Per), a SB with a systemic velocity of $12.2 \mathrm{~km} \mathrm{~s}^{-1}$ (Sect. 4.7), is most likely a nonmember. According to their spectral types and radial velocities, HD 23060 and 24640 are most likely members. HD 281159 is possibly a SB and could be a member (Sect. 4.8).

The remaining five B52 members are - by definition - Z99 non-members: HIP 16203, 16518, 17631, 18350, and 18614 (HD 21483, 21856, 23478, 24534, and 24912).

Both HD 21483 and 21856 were rejected by Z99. From the radial velocities, we conclude that HD 21483 is clearly a nonmember (this object also has a deviating position on the sky). However, HD 21856 could be a member, certainly given its early B1V spectral type, although its parallax is "small" (1.99 \pm $0.82 \mathrm{mas})$, its radial velocity is "large" $\left(29.7 \pm 3.3 \mathrm{~km} \mathrm{~s}^{-1}\right)$, and its (Hipparcos and Tycho-2) proper motion deviates from the mean of Per OB2.

HD 23478 (B3IV...) was not tested for membership in Z99 because the Hipparcos astrometric data quality indicator was large $(\mathrm{H} 30=3.21)$. Its OHP radial velocity $\left(15.8 \pm 4.0 \mathrm{~km} \mathrm{~s}^{-1}\right)$, its parallax $(4.19 \pm 1.03$ mas), and its Hipparcos and Tycho-2 proper motions are consistent with membership.

HD 24912 is $\xi$ Per, a celebrated run-away star (Blaauw 1961; Hoogerwerf et al. 2001). It moves away from Per OB2 with a relative radial velocity of $\sim 40 \mathrm{~km} \mathrm{~s}^{-1}$; we did not observe this object.

The last of the remaining B52 members is $\mathrm{X}$ Per (HD 24534). This O9.5pe high-mass X-ray binary was identified as member by Blaauw (1944, 1952), although its absolute magnitude gave rise to doubts on this classification. Z99 did not consider it because the Hipparcos proper motion was of insufficient quality, and for this reason we did not observe it. The reported systemic radial velocity is uncertain at the level of $50 \mathrm{~km} \mathrm{~s}^{-1}$ (B52; Wackerling 1972; Hutchings et al. 1975; Duflot et al. 1995), so membership remains uncertain.

\subsubsection{De Zeeuw et al. (1999) (Z99)}

We observed 14 stars in addition to 15 of the 17 B52 members (Sect. 5.2.1). All of these are certain or possible astrometric Per OB2 members from Z99 (see Sect. 2). Based on their OHP radial velocities, we conclude that HD 18830, 19216, 19567, 20113, 22114, and 23268 are unrelated field stars in the disk ("interlopers"). Based on their OHP radial velocities and the Hipparcos data, we conclude that HD 24012, 24583, 24970, and 26499 are members of Per OB2. We briefly discuss the remaining four stars below.

HD 20987: the OHP radial velocity $\left(-22.0 \mathrm{~km} \mathrm{~s}^{-1}\right)$ suggests that this Hipparcos acceleration binary is a background field star. This suggestion is strengthened by (i) the star's position on the sky $\sim 10^{\circ}$ away from the main body of the association; (ii) its relatively small parallax (1.80 \pm 1.08 mas); (iii) its early spectral type (B2V) combined with its relatively faint magnitude $(V=7.87 \mathrm{mag})$.

HD 278942: this faint object is a Hipparcos component binary (Table 1). Cernis (1993) identified HD 278942 as a "possible photometric B3III + F5I binary". Its presence in the Per OB2 cloud and its parallax $(4.83 \pm 1.21$ mas $)$ make it clear that the object is roughly at the same distance as Per OB2 $(\sim 318 \mathrm{pc}$; Z99). We find a radial velocity of $\sim 38 \mathrm{~km} \mathrm{~s}^{-1}$ for a B5 spectral type (SIMBAD/AGK3) and of $\sim 31 \mathrm{~km} \mathrm{~s}^{-1}$ for a B3III spectral type (Cernis 1993; see Sect. 4.8 for details). The uncertain spectral type, combined with duplicity, introduces a relatively large uncertainty in the inferred radial velocity. We suspect that this object belongs to Per OB2.

HD 23597: although the OHP radial velocity is small $\left(\sim 16 \mathrm{~km} \mathrm{~s}^{-1}\right)$, there is no compelling reason to believe it is not a member of Per OB2.

HD 23802: this B5Vn star is a Hipparcos component binary with a parallax of $3.09 \pm 1.21$ mas. At first sight, the OHP radial velocity $\left(\sim-50 \mathrm{~km} \mathrm{~s}^{-1}\right)$ seems inconsistent with membership. The three OHP exposures, however, give a relatively large spread in the inferred radial velocity $\left(\sim 12 \mathrm{~km} \mathrm{~s}^{-1}\right)$, which, if significant, might be interpreted as resulting from duplicity. This in turn could imply that the object does belong to Per OB2. Indirect evidence in favour of the above arguments is provided by the highly negative value of the OHP radial velocity: the correspondingly inferred distance of the object $(>4 \mathrm{kpc})$ is inconsistent with its spectral type and magnitude. 


\subsubsection{Literature radial velocities}

The analysis in Sects. 5.2.1 and 5.2.2 leaves three Z99 certain members which we did not observe, but for which radial velocities are available from SIMBAD and BvA. The measurement for HD 23244 (A0) is highly uncertain $\left(v_{\mathrm{rad}}=15 \pm 12.4 \mathrm{~km} \mathrm{~s}^{-1}\right.$ from SIMBAD), but is formally consistent with membership. HD 20825 (G5III) is a clear non-member at $6.2 \pm 1.2 \mathrm{~km} \mathrm{~s}^{-1}$. HD 281157 (B3V; a Hipparcos component binary) has a radial velocity of $20.9 \pm 2.1 \mathrm{~km} \mathrm{~s}^{-1}$ from BvA, and hence is a member.

\subsubsection{Conclusion on membership}

It follows from Sects. 5.2.1 and 5.2.2 that some of the classical/possible members are in fact field stars. This shows that the proper motion selection, while good, is not sufficient (this statement is particularly true for Perseus OB2 as the relative motion of the group is mostly along the radial direction/line of sight). Indeed, Z99 estimated the fraction of interlopers in their list of Hipparcos members, and concluded that for Per OB2 at 318 pc, this is $70-100 \%$ over the range $\mathrm{B} 9, \mathrm{~A}$ and later. These numbers agree with the Tycho- 2 membership analysis of Belikov et al. (2002b) and also with the findings of this investigation.

Table 7 summarizes our membership assignment for Per OB2, based on proper motions (B52, Z99), radial velocities (mostly this work), and photometry (Sect. 5.4). Membership derived from radial velocity data (Col. 5) is based on a $\pm 2 \sigma$ criterion. The remaining stars are, by definition, classified as non-members, with the exception of the following five "special cases", all of which are provisionally classified as possible members: HD 23802 and 281159, two suspected (spectroscopic) binaries, HD 278942, a faint target with an uncertain spectral type and low-quality spectra, and HD 24534 and 23244 , which have highly uncertain literature radial velocities. Final membership (Col. 6) is based on combining Hipparcos/astrometric (Z99) and radial velocity membership (Cols. 4 and 5) following the logical scheme Col. 4+Col. $5 \longrightarrow$ Col. 6, where $C+C \longrightarrow C, P+P \longrightarrow P, N+N \longrightarrow N$, $C+P \longrightarrow P, C+N \longrightarrow N$ (astrometric interloper), $P+C \longrightarrow C$ (astrometric binary), $P+N \longrightarrow N, N+C \longrightarrow C$ (astrometric binary), $N+P \longrightarrow N$ (combination not present); $\mathrm{C}, \mathrm{N}$, and $\mathrm{P}$ stand for certain, non-, and possible member. In practice, final membership in our sample is effectively the same as radial velocity membership.

\subsection{Internal structure}

B52 did not distinguish subgroups for Per OB2, although he did find subgroups for several other OB associations (cf. Blaauw 1964). However, Blaauw speculated that subgroups would be created as the Per OB2 association would evolve. Mirzoyan et al. (1999) claimed to have found substructure and expansion in Per OB2 with the Hipparcos analysis of 17 bright stars in the association. Mirzoyan, using Hipparcos Input Catalogue (HIC) radial velocities, found two "subgroups": one centered around $+17.4 \mathrm{~km} \mathrm{~s}^{-1}$ and one around $+26 \mathrm{~km} \mathrm{~s}^{-1}$. Belikov et al. (2002b) also presented evidence for two subgroups. We see no evidence for subdivision of Per OB2 in Fig. 8, but our sample is
Table 7. Membership and photometry of Per OB2. We first list the 29 objects observed by us (cf. Table 1). Below the horizontal line, we added two classical members from B52 that were not observed (Sect. 5.2.1) and three members from Z99 for which literature radial velocities exist (Sect. 5.2.3). Columns: (1) HD number; (2) spectral type; (3) classical membership assignment (B52; C: certain member; P: possible member; N: non-member); (4) Hipparcos membership (Z99); (5) membership based on radial velocity (mostly this work); (6) final membership, based on Cols. 4 and 5; in the case labeled with an asterisk, also photometric information was used; (7) Johnson $B$ magnitude (from Tycho); (8) Johnson $V$ magnitude (from Tycho); and (9) visual extinction $A_{V}$ from Strömgren or Johnson photometry (Sect. 5.4). We do not list photometric data for eight clear non-members (Sect. 5.4). Membership for the run-away star $\xi$ Per (HD 24912) comes down to semantics; we have indicated it by the label R.

\begin{tabular}{|c|c|c|c|c|c|c|c|c|}
\hline \multirow{2}{*}{$\begin{array}{r}\mathrm{HD} \\
\text { (1) }\end{array}$} & \multirow{2}{*}{$\begin{array}{l}\text { SpT } \\
\text { (2) }\end{array}$} & \multicolumn{4}{|c|}{ Membership } & \multirow{2}{*}{$\begin{array}{r}B \\
\text { (7) }\end{array}$} & \multirow{2}{*}{$\begin{array}{r}V \\
(8)\end{array}$} & \multirow{2}{*}{$\begin{array}{l}A_{V} \\
\text { (9) }\end{array}$} \\
\hline & & (3) & (4) & (5) & (6) & & & \\
\hline 18830 & $\mathrm{~A} 0$ & & $\mathrm{C}$ & $\mathrm{N}$ & $\mathrm{N}$ & & & \\
\hline 19216 & B9V & & $\mathrm{P}$ & $\mathrm{N}$ & $\mathrm{N}$ & & & \\
\hline 19567 & B9 & & $\mathrm{C}$ & $\mathrm{N}$ & $\mathrm{N}$ & & & \\
\hline 20113 & B8 & & $\mathrm{P}$ & $\mathrm{N}$ & $\mathrm{N}$ & & & \\
\hline 20987 & $\mathrm{~B} 2 \mathrm{~V}$ & & $\mathrm{C}$ & $\mathrm{N}$ & $\mathrm{N}$ & & & \\
\hline 21483 & B3III & $\mathrm{P}$ & $\mathrm{N}$ & $\mathrm{N}$ & $\mathrm{N}$ & & & \\
\hline 21856 & B1V & $\mathrm{C}$ & $\mathrm{N}$ & $\mathrm{C}$ & $\mathrm{C}$ & 5.816 & 5.899 & 0.569 \\
\hline 22114 & $\mathrm{~B} 8 \mathrm{Vp}$ & & $\mathrm{P}$ & $\mathrm{N}$ & $\mathrm{N}$ & & & \\
\hline 22951 & B $0.5 \mathrm{~V}$ & $\mathrm{C}$ & $\mathrm{C}$ & $\mathrm{C}$ & $\mathrm{C}$ & 4.927 & 4.975 & 0.737 \\
\hline 23060 & $\mathrm{~B} 2 \mathrm{Vp}$ & $\mathrm{C}$ & $\mathrm{P}$ & $\mathrm{C}$ & $\mathrm{C}$ & 7.585 & 7.531 & 1.072 \\
\hline 23180 & B1III & $\mathrm{P}$ & $\mathrm{P}$ & $\mathrm{N}$ & $\mathrm{N}$ & 3.871 & 3.855 & 0.886 \\
\hline 23268 & A0 & & $\mathrm{C}$ & $\mathrm{N}$ & $\mathrm{N}$ & & & \\
\hline 23478 & B3IV... & $\mathrm{C}$ & & $\mathrm{C}$ & $\mathrm{C}$ & 6.717 & 6.688 & 0.783 \\
\hline 23597 & B8 & & $\mathrm{C}$ & $\mathrm{C}$ & $\mathrm{C}$ & 8.262 & 8.225 & 0.795 \\
\hline 23625 & $\mathrm{~B} 2.5 \mathrm{~V}$ & $\mathrm{C}$ & $\mathrm{C}$ & $\mathrm{C}$ & $\mathrm{C}$ & 6.598 & 6.564 & 0.852 \\
\hline 23802 & B5Vn & & $\mathrm{C}$ & $\mathrm{P}$ & $\mathrm{P}$ & 7.555 & 7.386 & 1.029 \\
\hline 24012 & B5 & & $\mathrm{C}$ & $\mathrm{C}$ & $\mathrm{C}$ & 7.835 & 7.850 & 0.632 \\
\hline 24131 & B1V & $\mathrm{C}$ & $\mathrm{C}$ & $\mathrm{C}$ & $\mathrm{C}$ & 5.747 & 5.784 & 0.737 \\
\hline 24190 & $\mathrm{~B} 2 \mathrm{~V}$ & $\mathrm{C}$ & $\mathrm{C}$ & $\mathrm{C}$ & $\mathrm{C}$ & 7.458 & 7.449 & 0.936 \\
\hline 24398 & B1Ib & $\mathrm{C}$ & $\mathrm{C}$ & $\mathrm{C}$ & $\mathrm{C}$ & 2.966 & 2.883 & 1.158 \\
\hline 24583 & B7V & & $\mathrm{P}$ & $\mathrm{C}$ & $\mathrm{C}$ & 9.047 & 9.002 & 0.626 \\
\hline 24640 & $\mathrm{~B} 1.5 \mathrm{~V}$ & $\mathrm{C}$ & $\mathrm{P}$ & $\mathrm{C}$ & $\mathrm{C}$ & 5.431 & 5.489 & 0.563 \\
\hline 24970 & A0 & & $\mathrm{P}$ & $\mathrm{C}$ & $\mathrm{N}^{*}$ & 7.589 & 7.466 & 0.643 \\
\hline 25539 & B3V & $\mathrm{C}$ & $\mathrm{C}$ & $\mathrm{C}$ & $\mathrm{C}$ & 6.873 & 6.874 & 0.762 \\
\hline 25799 & B3V... & $\mathrm{C}$ & $\mathrm{C}$ & $\mathrm{C}$ & $\mathrm{C}$ & 7.066 & 7.032 & 0.802 \\
\hline 25833 & B5V:p & $\mathrm{C}$ & $\mathrm{C}$ & $\mathrm{C}$ & $\mathrm{C}$ & 6.710 & 6.720 & 0.571 \\
\hline 26499 & B9 & & $\mathrm{C}$ & $\mathrm{C}$ & $\mathrm{C}$ & 9.205 & 9.057 & 1 \\
\hline 278942 & B3III & & $\mathrm{C}$ & $\mathrm{P}$ & $\mathrm{P}$ & 10.307 & 9.175 & 4.750 \\
\hline 281159 & B5V & $\mathrm{C}$ & $\mathrm{P}$ & $\mathrm{P}$ & $\mathrm{P}$ & 9.278 & 8.681 & 2.720 \\
\hline 24534 & O9.5pe & $\bar{C}$ & & $\bar{P}$ & $\bar{P}$ & 6.862 & 6.793 & 1.569 \\
\hline 24912 & O7.5Iab: & $\mathrm{P}$ & $\mathrm{N}$ & $\mathrm{N}$ & $\mathrm{R}$ & 4.022 & 4.042 & 0.859 \\
\hline 20825 & G5III & & $\mathrm{C}$ & $\mathrm{N}$ & $\mathrm{N}$ & & & \\
\hline 23244 & A0 & & $\mathrm{C}$ & $\mathrm{P}$ & $\mathrm{P}$ & & & \\
\hline 281157 & B3V & & $\mathrm{C}$ & $\mathrm{C}$ & $\mathrm{C}$ & 9.811 & 9.177 & 2.930 \\
\hline
\end{tabular}

small. For the particular case of Per OB2, Hipparcos astrometric radial velocities offer no viable alternative to study internal structure issues: as the relative motion of the association is mostly along the line of sight, Hipparcos astrometric radial velocities are expected to be accurate to $\sim 2.5 \mathrm{~km} \mathrm{~s}^{-1}$ 

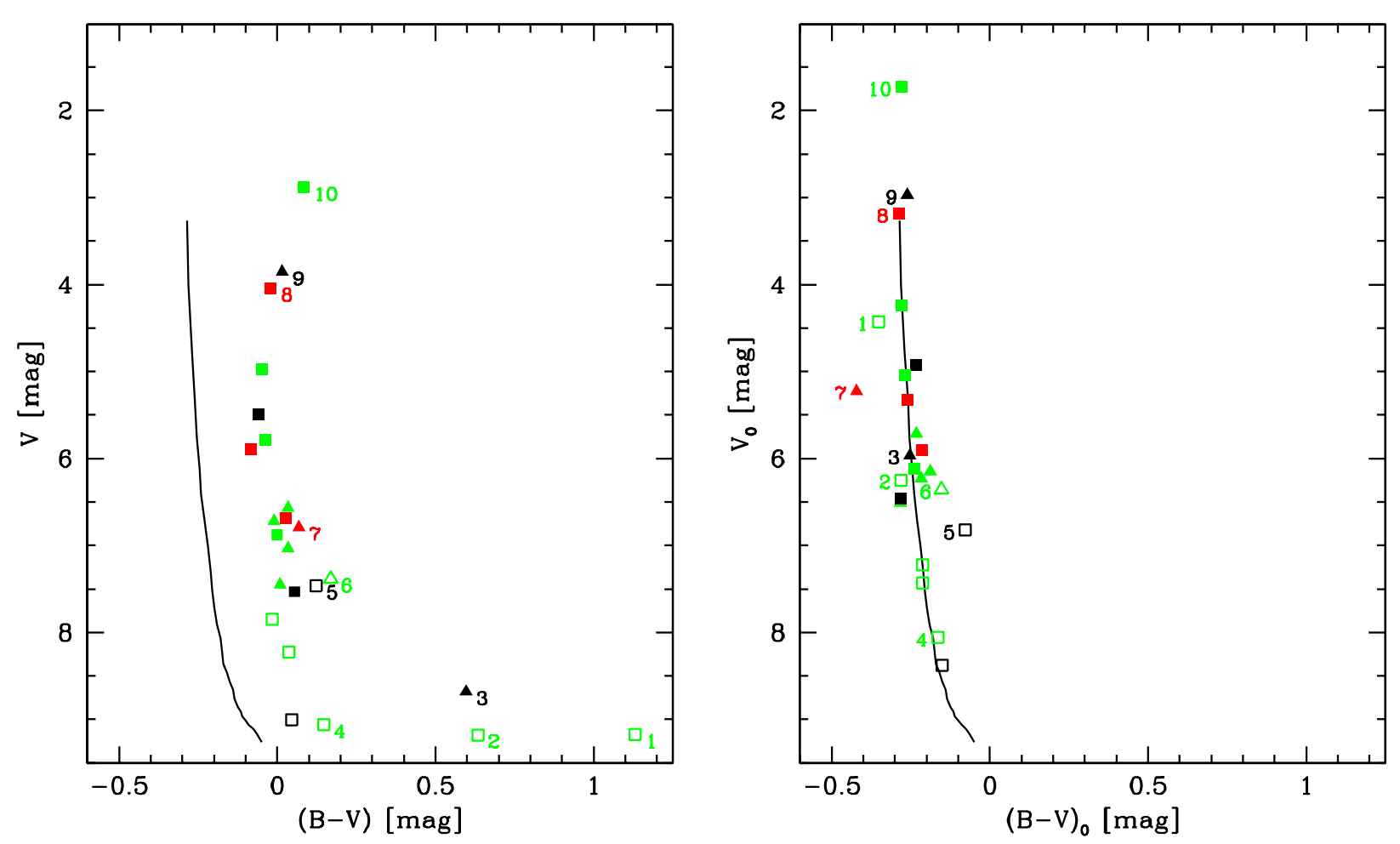

Fig. 9. Observed (left) and de-reddened (right) colour-magnitude diagram of Per OB2 $\left(R_{V}=3.2\right)$. The curves represent the zero-age main sequence (Schmidt-Kaler 1982) at the mean distance of Per OB2 (318 pc; Z99). Symbols have the same meaning as in Fig. 7, except that two new possible SBs have been given triangular shapes: HD 23802 (labeled 6) and HD 281159 (labeled 3). Numerical labels (HD numbers): $1=$ 278942; 2 = 281157; $3=281159 ; 4=26499 ; 5=24970 ; 6=23802 ; 7=24534$ (X Per); $8=24912$ (the run-away $\xi$ Per); $9=23180$ (o Per); $10=24398$ ( $\zeta$ Per). See Sect. 5.4 for details.

(Dravins et al. 1999), similar to the accuracy of the spectroscopic radial velocities presented here.

\subsection{Colour-magnitude diagram}

Figure 9 shows the observed and de-reddened colourmagnitude diagrams (CMDs). In order to construct these, we removed the eight non-members identified above the horizontal line in Table 7 (denoted by "N" in Col. 6), but retained HD 24970 as this is a possible member based on the astrometry and radial velocity measurement. We added the two classical members from B52 that we did not observe ( $\xi$ Per/HD 24912, which is a "member" in some sense, and X Per/HD 24534, which is a doubtful member; Sect. 5.2.1) as well as HD 281157 which we classified as member based on a literature radial velocity (Sect. 5.2.3). We retrieved Johnson $B$ and $V$ magnitudes for the resulting 24 stars and derived individual extinctions $A_{V}$ from both Strömgren and Johnson photometry using $R_{V}=3.2$. We adopted $A_{V}=1 \mathrm{mag}$, roughly the mean extinction for Per OB2 (e.g., Belikov et al. 2002b), for the star HD 26499 which lacked appropriate photometry; this star is labeled "4" in Fig. 9.

The right panel of Fig. 9 displays a tight main sequence. The stars HD 278942 and 281157 (labels "1" and "2", respectively) both suffer from large extinctions $\left(A_{V}=4.75\right.$ and $2.93 \mathrm{mag}$, respectively). The accuracy of our simple extinction correction is such that the de-reddened CMD locations are fully consistent with membership. The locations of two B5V stars with "peculiar"/deviating radial velocities (HD 281159 with $v_{\text {rad }}=8.4 \mathrm{~km} \mathrm{~s}^{-1}$ and HD 23802 with $v_{\text {rad }}=$ $-52.3 \mathrm{~km} \mathrm{~s}^{-1}$, labels "3" and "6", respectively) are also consistent with membership, which suggests these stars could be multiple. Our adoption of the average extinction to Per OB2 for HD 26499 (label "4", see above) puts it indeed on the dereddened main sequence.

The CMD location of HD 24970 (label "5") raises doubt about its physical association with Per OB2. Although the observed radial velocity $\left(23.2 \pm 2.2 \mathrm{~km} \mathrm{~s}^{-1}\right)$ is consistent with membership, we suspect that, given the relatively bright magnitude for its spectral type (A0), its relatively "large" Hipparcos parallax (4.95 \pm 0.99 mas), and given the fact that this star is not a certain but "only" a possible Z99 member, it is in fact a foreground object (we found $A_{V}=0.64 \mathrm{mag}$, roughly the foreground extinction to Per OB2; Rydgren 1971). The peculiar location of X Per can be ascribed to the emission lines in its spectrum. The locations of the runaway star $\xi$ Per (label "8") and the supergiant $\zeta$ Per (label "10") are consistent with membership. Finally, o Per (label "9") seems to fit the zero-age main sequence, even though its radial velocity indicates it is a non-member (the object is, nonetheless, located in the Perseus molecular cloud).

While our radial velocity study has removed some interlopers from earlier member lists of Per OB2, including HD 21483 (B3III) and o Per (B1III), most of these have mid-B spectral 
types or later. As a result, the location of the main sequence turn off is unchanged, and so are the age determinations based on this.

\section{Concluding remarks}

We have presented radial velocity measurements derived from high-resolution spectroscopy for 29 candidate B- and A-type members of the association Per OB2 obtained with AURELIE at Observatoire de Haute-Provence. The radial velocities were found via cross correlation with the spectra of observed standard stars with known radial velocities. A careful observing strategy and data reduction, rigorous removal of suspect exposures caused by an unstable detector, and use of multiple standard stars to minimize template mismatch, resulted in derived radial velocities accurate to $\sim 2-3 \mathrm{~km} \mathrm{~s}^{-1}$. We have checked our error estimates by comparison with literature measurements.

The new measurements, together with those for some additional candidate members from earlier studies, show that Per OB2 lies offset by $\sim 15 \mathrm{~km} \mathrm{~s}^{-1}$ from the $v_{\text {rad }}$ distribution for field stars in this direction. The dispersion amongst the measurements for the Per OB2 members is only $3.9 \mathrm{~km} \mathrm{~s}^{-1}$, which provides an upper limit to our radial velocity accuracy. The internal one-dimensional velocity dispersion of the association is smaller than we can measure, but is likely to be $\sim 1-3 \mathrm{~km} \mathrm{~s}^{-1}$.

The radial velocity measurements confirm many of the classical astrometric members, but not all. Some earlier "secure" members turn out to have discrepant $v_{\text {rad }}$ values; this means they are either previously unknown spectroscopic binaries or are genuine non-members. The bulk of these discrepant stars have $v_{\text {rad }}$ of about $6 \mathrm{~km} \mathrm{~s}^{-1}$, which puts them right at the velocity of the disk (field) stars in this direction, suggesting they are non-members. Removing these from the sample results in a very narrow main sequence in the colour-magnitude diagram. The two outliers in this diagram are the X-ray binary $\mathrm{X}$ Per, whose membership remains uncertain, and the A0 star HD 24970. Even though its radial velocity and proper motion are consistent with the space motion of Per OB2, we conclude HD 24970 is a foreground object.

The classical study by BvA had already shown that a significant fraction of the B-type members of Per OB2 are spectroscopic binaries. Our study has identified HD 23802, HD 281159, and possibly also the peculiar object HD 278942, as additional candidate spectroscopic binaries. It would be worthwhile to obtain multi-epoch spectroscopy for all the Btype members of Per OB2, in order to derive accurate orbits for the spectroscopic binaries, and possibly identify additional ones. The effort required is significant, but would be invaluable in determining the present day mass function of the association, and the nature of the binary population.

The modest accuracy of the Hipparcos parallaxes and proper motions for groups such as Per OB2, combined with the relatively bright limiting magnitude of the Hipparcos Catalogue, did not allow reliable identification of members later than $\sim \mathrm{B} 9-\mathrm{A} 0$, even though these are no doubt present (see, e.g., Preibisch et al.'s 2002 study of the Sco-Cen OB association). A first step to probe membership of Per OB2 for later spectral types was made by Belikov et al. (2002a,b), who constructed a list of $\sim 1000$ possible members of Per OB2 based on Tycho-2 proper motions and photometric information. Our results demonstrate that a natural next step to distinguish members from interlopers in this list would be to obtain spectra from which to derive radial velocities (as well as spectral types and luminosity classes).

ESA's future astrometry satellite GAIA (Perryman et al. 2001) will extend proper motion studies to much fainter limiting magnitude than Hipparcos, and will allow kinematic identification of low-mass members, including all pre-main sequence objects, in Per OB2 and other nearby associations. GAIA will also allow study of more distant groups in unprecedented detail. Besides astrometry, GAIA will also collect multi-epoch spectroscopic measurements for all stars down to $V \sim 17 \mathrm{mag}$. However, due to the specifics of the wavelength range used $(\sim 250 \AA$ around the CaII-triplet near $860 \mathrm{~nm})$, the resulting radial velocities for early-type stars will likely be of modest precision. The currently planned ground-based all-sky multi-object spectroscopic radial-velocity survey RAVE uses the same spectral range, and may hence suffer from the same limitation. Our study shows that dedicated (multi-epoch) spectroscopic studies of the early-type stars in the nearby associations are feasible, and are crucial in determining membership and the nature of the population of high-mass stars in these young stellar groups.

Acknowledgements. It is a pleasure to thank Adriaan Blaauw, Anthony Brown, Huib Henrichs, Lex Kaper, and Werner Verschueren for many fruitful discussions, and the referee, Jon Morse, for helpful comments. The observations presented here were obtained at the Observatoire de Haute-Provence (CNRS) in France. This research has made use of the ADS (NASA) and SIMBAD (CDS) services and the IRAF Data Reduction and Analysis System. IRAF is distributed by the National Optical Astronomy Observatories, which are operated by the Association of Universities for Research in Astronomy, Inc., under cooperative agreement with the National Science Foundation.

\section{References}

Abt, H. A., \& Levy, S. G. 1978, ApJS, 36, 241

Abt, H. A. 1985, ApJS, 59, 95

Andersson, B.-G., Wannier, P. G., Moriarty-Schieven, G. H., \& Bakker, E. J. 2000, AJ, 119, 1325

Barbier-Brossat, M., \& Figon, P. 2000, A\&AS, 142, 217

Belikov, A. N., Kharchenko, N. V., Piskunov, A. E., et al. 2002a, A\&A, 384, 145

Belikov, A. N., Kharchenko, N. V., Piskunov, A. E., Schilbach, E., \& Scholz, R.-D. 2002b, A\&A, 387, 117

Blaauw, A. 1944, Bull. Astron. Inst. The Netherlands, 10, 29

Blaauw, A. 1952, Bull. Astron. Inst. The Netherlands, 11, 405 (B52)

Blaauw, A. 1961, Bull. Astron. Inst. The Netherlands, 15, 265

Blaauw, A. 1964, ARA\&A, 2, 213

Blaauw, A., \& van Albada, T. S. 1963, ApJ, 137, 791 (BvA)

Blaauw, A., \& van Hoof, A. 1963, ApJ, 137, 821

Brown, A. G. A., Dekker, G., \& de Zeeuw, P. T. 1997, MNRAS, 285, 479

de Bruijne, J. H. J. 1999, MNRAS, 306, 381

Cernis, K. 1993, Baltic Astron., 2, 214

Dravins, D., Lindegren, L., \& Madsen, S. 1999, A\&A, 348, 1040

Duflot, M., Fehrenbach, C., Mannone, C., Burnage, R., \& Genty, V. 1995, A\&AS, 110, 177 
ESA 1997, The Hipparcos and Tycho Catalogues, ESA SP-1200 Evans, D. S. 1967, IAUS, 30, 57

Fekel, F. C. 1985, in Stellar Radial Velocities, IAU Coll., 88, 335

Fekel, F. C. 1990, private communication in Morse et al. (1991)

Gies, D. R., \& Bolton, C. T. 1986, ApJS, 61, 419

Gillet, D., Burnage, R., Kohler, D., et al. 1994, A\&AS, 108, 181

Giménez, A., \& Clausen, J. V. 1994, A\&A, 291, 795

Grenier, S., Baylac, M.-O., Rolland, L., et al. 1999, A\&AS, 137, 451

Griffin, R. E. M., David, M., \& Verschueren, W. 2000, A\&AS, 147, 299

Guetter, H. H. 1977, AJ, 598

Hoogerwerf, R., \& Aguilar, L. A. 1999, MNRAS, 306, 394

Hoogerwerf, R., de Bruijne, J. H. J., \& de Zeeuw, P. T. 2001, A\&A, 365,49

Hutchings, J. B., Crampton, D., \& Redman, R. O. 1975, MNRAS, 170,313

Jaschek, C., \& Jaschek, M. 1990, The classification of stars (CUP, Cambridge)

Latham, D. W., \& Stefanik, R. P. 1982, Trans. IAU XXIB, 269

Lennon, D. J., Dufton, P. L., \& Fitzsimmons, A. 1992, A\&AS, 94, 569

Liu, T., Janes, K. A., \& Bania, T. M. 1989, AJ, 98, 626

Lucy, L. B., \& Sweeney, M. A. 1971, AJ, 76, 544

Mirzoyan, L. V., Hambaryan, V. V., Akopian, A. A., Poghosyan, A. V., \& Salukvadze, G. N. 1999, Astrofizika, 42, 333

Moore, J. H. 1932, A general catalogue of the radial velocities of stars, nebulae and clusters, Publ. Lick Obs., 18, 1

Morris, S. L., Bolton, C. T., Fernie, J. D., \& Percy, J. R. 1988, PASP, 100,954

Morse, J. A., Mathieu, R. D., \& Levine, S. E. 1991, AJ, 101, 1495
Perryman, M. A. C., de Boer, K. S., Gilmore, G., et al. 2001, A\&A, 369, 339

Petrie, R. M., \& Pearce, J. A. 1961, Publ. Dominion Astrophys. Obs., 12,1

Popper, D. M. 1974, ApJ, 188, 559

Popper, D. M., \& Hill, G. 1991, AJ, 101, 600

Preibisch, T., Brown, A. G. A., Bridges, T., Günther, E., \& Zinnecker, H. 2002, AJ, 124, 404

Roman, N. G. 1978, AJ, 83, 172

Rydgren, A. E. 1971, PASP, 83, 656

Schmidt-Kaler, Th. 1982, in Landolt-Börnstein, Group VI: A\&A, vol. 1, Sect. 5.2.2

Sonnentrucker, P., Foing, B. H., Breitfellner, M., \& Ehrenfreund, P. 1999, A\&A, 346, 936

Stickland, D. J., \& Lloyd, C. 1998, Observatory, 118, 138

Tody, D. 1993, in Astronomical data analysis software and systems II, ed. R. J. Hanisch, R. J. V. Brissenden, \& J. Barnes, ASP Conf. Ser., 52, 173

Verschueren, W., Brown, A. G. A., Hensberghe, H., et al. 1997, PASP, 109,868

Verschueren, W., \& David, M. 1999, A\&AS, 136, 591

Verschueren, W., David, M., \& Griffin, R. E. M. 1999, A\&AS, 140, 107

Wackerling, L. R. 1972, PASP, 84, 827

Wilson, R. E., \& Joy, A. H. 1952, ApJ, 115, 157

Wolff, S. C. 1978, ApJ, 222, 556

de Zeeuw, P. T., Hoogerwerf, R., de Bruijne, J. H. J., Brown, A. G. A., \& Blaauw, A. 1999, AJ, 117, 354 (Z99)

Zentelis, N. 1983, A\&AS, 53, 445 (Z83) 